\title{
Calculus of Variations with Differential Forms
}

\author{
Saugata Bandyopadhyay Bernard Dacorogna Swarnendu Sil
}

\begin{abstract}
We study integrals of the form $\int_{\Omega} f(d \omega)$, where $1 \leq k \leq n, f: \Lambda^{k} \rightarrow \mathbb{R}$ is continuous and $\omega$ is a $(k-1)$-form. We introduce the appropriate notions of convexity, namely ext. one convexity, ext. quasiconvexity and ext. polyconvexity. We study their relations, give several examples and counterexamples. We finally conclude with an application to a minimization problem.
\end{abstract}

Keywords. calculus of variations, differential forms, quasiconvexity, polyconvexity and ext. one convexity

\section{Introduction}

In this article, we study integrals of the form

$$
\int_{\Omega} f(d \omega)
$$

where $1 \leq k \leq n$ are integers, $f: \Lambda^{k} \rightarrow \mathbb{R}$ is a continuous function and $\omega$ is a $(k-1)$ form. When $k=1$, by abuse of notations identifying $\Lambda^{1}$ with $\mathbb{R}^{n}$ and the operator $d$ with the gradient, this is the classical problem of the calculus of variations where one studies integrals of the form

$$
\int_{\Omega} f(\nabla \omega)
$$

This is a scalar problem in the sense that there is only one function $\omega$. It is well known that in this last case the convexity of $f$ plays a crucial role. As soon as $k \geq 2$, the problem is more of a vectorial nature, since then $\omega$ has now several components. However, it has

S. Bandyopadhyay: IISER Kolkata, Mohanpur Campus, Mohanpur-741252, India; e-mail: saugata.bandyopadhyay@iiserkol.ac.in

B. Dacorogna: Section de Mathématiques, EPFL, 1015 Lausanne, Switzerland; e-mail: bernard.dacorogna@epfl.ch

S. Sil: Section de Mathématiques, EPFL, 1015 Lausanne, Switzerland; e-mail: swarnendu.sil@epfl.ch

Mathematics Subject Classification (2010): Primary 49-XX 
some special features that a general vectorial problem does not have. Before going further one should have two examples in mind.

1) If $k=2$, with our usual abuse of notations,

$$
\omega: \mathbb{R}^{n} \rightarrow \mathbb{R}^{n} \text { and } \quad d \omega=\operatorname{curl} \omega .
$$

2) If $k=n$, by abuse of notations and up to some changes of signs,

$$
\omega: \mathbb{R}^{n} \rightarrow \mathbb{R}^{n} \text { and } \quad d \omega=\operatorname{div} \omega .
$$

So let us now discuss some specific features of our problem.

- The first important point is the lack of coercivity. Indeed, even if the function $f$ grows at infinity as the norm to a certain power, this does not imply control on the full gradient but only on some combination of it, namely $d \omega$. So when looking at minimization problems, this fact requires special attention (see Theorem 5.1).

- From the point of view of convexity, the situation is, in some cases, simpler than in the general vectorial problem. Indeed consider the above two examples (with $n=3$ for the first one). Although the problems are vectorial they behave as if they were scalar (cf. Theorem 2.8).

- One peculiarity (cf. Theorem 3.3) that particularly stands out is how the problem changes its behaviour with a change in the order of the form. When $k$ is odd, or when $2 k>n$ (in particular $k=n$ ), there is no nonlinear function which is ext. quasiaffine and therefore, the problem behaves as if it were scalar. However, the situation changes significantly when $k \leq 2 n$ is even. It turns out that we have an ample supply of nonlinear functions that are ext. quasiaffine in this case. For example, the nonlinear function

$$
f(\xi)=\langle c ; \xi \wedge \xi\rangle
$$

where $c \in \Lambda^{2 k}$, is ext. quasiaffine. See Theorem 3.3 for the complete characterization of ext. quasiaffine functions which, in turn, determines all weakly continuous functions with respect to the $d$-operator, see Bandyopadhyay-Sil [4] for detail.

Because of the special nature of our problem, we are led to introduce the following terminology: ext. one convexity, ext. quasiconvexity and ext. polyconvexity, which are the counterparts of the classical notions of the vectorial calculus of variations (see, in particular Dacorogna [8]), namely rank one convexity, quasiconvexity and polyconvexity. The relations between these notions (cf. Theorem 2.8) as well as their manifestations on the minimization problem are the subject of the present paper. Examples and counterexamples are also discussed in details, notably the case of ext. quasiaffine functions (see Theorem 3.3), the quadratic case (see Theorem 4.5) and a fundamental counterexample (see Theorem 4.8) similar to the famous example of Sverak [20]. 
Some of what has been done in this article may also be seen through classical vectorial calculus of variations. This connection is elaborated and pursued in detail in a forthcoming article, see Bandyopadhyay-Sil [3]. However, the case of differential forms in the context of calculus of variations deserves a separate and independent treatment because of its special algebraic structure which renders much of the calculation intrinsic, natural and coordinate free.

We conclude this introduction by pointing out that the results discussed in this introduction may be interpreted very broadly in terms of the theory of compensated compactness introduced by Murat and Tartar, see [14], [21] and see also Dacorogna [7], RobbinRogers-Temple [15]. In particular, our notion of ext. one convexity is related to the so called condition of convexity in the directions of the wave cone $\Lambda$. Our definition of ext. quasiconvexity is related to those of $A$ and $A-B$ quasiconvexity introduced by Dacorogna (cf. [6] and [7]), see also Fonseca-Müller [10].

\section{Definitions and main properties}

\subsection{Definitions}

We start with the different notions of convexity and affinity.

Definition 2.1. Let $1 \leq k \leq n$ and $f: \Lambda^{k} \rightarrow \mathbb{R}$.

(i) We say that $f$ is ext. one convex, if the function

$$
g: t \rightarrow g(t)=f(\xi+t \alpha \wedge \beta)
$$

is convex for every $\xi \in \Lambda^{k}, \alpha \in \Lambda^{k-1}$ and $\beta \in \Lambda^{1}$. If the function $g$ is affine we say that $f$ is ext. one affine.

(ii) $f$ is said to be ext. quasiconvex, if $f$ is Borel measurable, locally bounded and

$$
\int_{\Omega} f(\xi+d \omega) \geq f(\xi) \text { meas } \Omega,
$$

for every bounded open set $\Omega \subset \mathbb{R}^{n}, \xi \in \Lambda^{k}$ and $\omega \in W_{0}^{1, \infty}\left(\Omega ; \Lambda^{k-1}\right)$. If equality holds, we say that $f$ is ext. quasiaffine.

(iii) We say that $f$ is ext. polyconvex, if there exists a convex function

$$
F: \Lambda^{k} \times \Lambda^{2 k} \times \cdots \times \Lambda^{[n / k] k} \rightarrow \mathbb{R}
$$

such that

$$
f(\xi)=F\left(\xi, \xi^{2}, \cdots, \xi^{[n / k]}\right), \text { for all } \xi \in \Lambda^{k} .
$$

If $F$ is affine, we say that $f$ is ext. polyaffine. 
Remark 2.2. (i) The ext. stands for exterior product in the first and third ones and for the exterior derivative for the second one.

(ii) When $k$ is odd (since then $\xi^{s}=0$ for every $s \geq 2$ ) or when $2 k>n$ (in particular, when $k=n$ or $k=n-1)$, then ext. polyconvexity is equivalent to ordinary convexity (see Proposition 2.14).

(iii) When $k=1$, all the above notions are equivalent to the classical notion of convexity (cf. Theorem 2.8).

(iv) As in Proposition 5.11 of [8], it can easily be shown that if the inequality of ext. quasiconvexity holds for a given bounded open set $\Omega$, it holds for any bounded open set.

(v) The definition of ext. quasiconvexity is equivalent (as in Proposition 5.13 of [8]) to the following. Let $D=(0,1)^{n}$, the inequality

$$
\int_{D} f(\xi+d \omega) \geq f(\xi)
$$

holds for every $\xi \in \Lambda^{k}$ and for every $\omega \in W_{\text {per }}^{1, \infty}\left(D ; \Lambda^{k-1}\right)$, where

$$
W_{\text {per }}^{1, \infty}\left(D ; \Lambda^{k-1}\right)=\left\{\omega \in W^{1, \infty}\left(D ; \Lambda^{k-1}\right): \omega \text { is 1-periodic in each variable }\right\} .
$$

Definition 2.3. Let $0 \leq k \leq n$ and $f: \Lambda^{k} \rightarrow \mathbb{R}$. The Hodge transform of $f$ is the function $f_{*}: \Lambda^{n-k} \rightarrow \mathbb{R}$ defined as,

$$
f_{*}(\xi)=f(* \xi), \text { for all } \xi \in \Lambda^{n-k} .
$$

The notion of Hodge transform allows us to extend the notions of convexity with respect to the interior product and the $\delta$-operator as follows.

Definition 2.4. Let $0 \leq k \leq n-1$ and $f: \Lambda^{k} \rightarrow \mathbb{R}$. We say that

(i) $f$ is int. one convex, if $f_{*}$ is ext. one convex.

(ii) $f$ is int. quasiconvex, if $f_{*}$ is ext. quasiconvex.

(iii) $f$ is int. polyconvex, if $f_{*}$ is ext. polyconvex.

Remark 2.5. (i) Statements similar to those in Remark 2.2 hold in the case of int. convexity as well.

(ii) It is easy to check that $f$ is int. one convex, if and only if the function

$$
g: t \rightarrow g(t)=f(\xi+t \beta\lrcorner \alpha)
$$

is convex for every $\xi \in \Lambda^{k}, \beta \in \Lambda^{1}$ and $\alpha \in \Lambda^{k+1}$. Furthermore, $f$ is int. quasiconvex if and only if $f$ is Borel measurable, locally bounded and

$$
\int_{\Omega} f(\xi+\delta \omega) \geq f(\xi) \text { meas } \Omega
$$

for every bounded open set $\Omega \subset \mathbb{R}^{n}, \xi \in \Lambda^{k}$ and $\omega \in W_{0}^{1, \infty}\left(\Omega ; \Lambda^{k+1}\right)$. The third one is however a little more involved and we leave out the details. 
In what follows, we will discuss the case of ext. convexity only. The case of int. convexity can be handled analogously.

\subsection{Preliminary lemmas}

In this subsection, we state two lemmas which will be used in sequel. See [17] for the proofs. We start with the following problem of prescribed differentials. Let us recall that $\alpha \in \Lambda^{k}$ is said to be 1 -divisible if $\alpha=a \wedge b$, for some $a \in \Lambda^{k-1}, b \in \Lambda^{1}$.

Lemma 2.6. Let $1 \leq k \leq n$ and let $\omega_{1}, \omega_{2} \in \Lambda^{k}$. Then, there exists $\omega \in W^{1, \infty}\left(\Omega ; \Lambda^{k-1}\right)$ satisfying

$$
d \omega \in\left\{\omega_{1}, \omega_{2}\right\}, \text { a.e. in } \Omega,
$$

(and taking both values), if and only if $\omega_{1}-\omega_{2}$ is 1-divisible.

Using Lemma 2.6, one can deduce the following approximation lemma for $k$-forms. See Lemma 3.11 of [8] for the case of the gradient.

Lemma 2.7. Let $1 \leq k \leq n, t \in[0,1]$ and let $\alpha, \beta \in \Lambda^{k}$ be such that $\alpha \neq \beta$ and $\alpha-\beta$ is 1-divisible. Let $\Omega \subset \mathbb{R}^{n}$ be open, bounded and let $\omega: \bar{\Omega} \rightarrow \Lambda^{k-1}$ satisfy

$$
d \omega=t \alpha+(1-t) \beta, \text { in } \bar{\Omega}
$$

Then, for every $\epsilon>0$, there exist $\omega_{\epsilon} \in \operatorname{Aff}_{\text {piece }}\left(\bar{\Omega} ; \Lambda^{k-1}\right)$ and disjoint open sets $\Omega_{\alpha}, \Omega_{\beta} \subset$ $\Omega$ such that

1. $\left|\operatorname{meas}\left(\Omega_{\alpha}\right)-t \operatorname{meas}(\Omega)\right| \leqslant \epsilon$ and $\left|\operatorname{meas}\left(\Omega_{\beta}\right)-(1-t) \operatorname{meas}(\Omega)\right| \leqslant \epsilon$,

2. $\omega_{\epsilon}=\omega$, in a neighbourhood of $\partial \Omega$,

3. $\left\|\omega_{\epsilon}-\omega\right\|_{L^{\infty}(\bar{\Omega})} \leqslant \epsilon$,

4. $d \omega_{\epsilon}(x)= \begin{cases}\alpha, & \text { if } x \in \Omega_{\alpha}, \\ \beta, & \text { if } x \in \Omega_{\beta},\end{cases}$

5. $\operatorname{dist}\left(d \omega_{\epsilon}(x) ;\{t \alpha+(1-t) \beta: t \in[0,1]\}\right) \leqslant \epsilon$, for all $x \in \Omega$ a.e.

\subsection{Main properties}

The different notions of convexity are related as follows.

Theorem 2.8. Let $1 \leq k \leq n$ and $f: \Lambda^{k} \rightarrow \mathbb{R}$.

(i) The following implications hold

$f$ convex $\Rightarrow f$ ext. polyconvex $\Rightarrow f$ ext. quasiconvex $\Rightarrow f$ ext. one convex. 
(ii) If $k=1, n-1, n$ or $k=n-2$ is odd, then

$$
f \text { convex } \Leftrightarrow f \text { ext. polyconvex } \Leftrightarrow f \text { ext. quasiconvex } \Leftrightarrow f \text { ext. one convex. }
$$

Moreover, if $k$ is odd or $2 k>n$, then

$$
f \text { convex } \Leftrightarrow f \text { ext. polyconvex. }
$$

(iii) If either $2 \leq k \leq n-3$ or $k=n-2 \geq 2$ is even, then

$$
\text { f ext. polyconvex } \underset{\nLeftarrow}{\Rightarrow} \text { ext. quasiconvex, }
$$

while if $2 \leq k \leq n-3$ (and thus $n \geq k+3 \geq 5$ ), then

$$
\text { f ext. quasiconvex } \underset{\nLeftarrow}{\Rightarrow} \text { ext. one convex. }
$$

Remark 2.9. (i) The last statement in (ii) for $k$ even and $n \geq 2 k$ is false, as the following simple example shows. Let $f: \Lambda^{2}\left(\mathbb{R}^{4}\right) \rightarrow \mathbb{R}$ be defined by

$$
f(\xi)=\left\langle e^{1} \wedge e^{2} \wedge e^{3} \wedge e^{4} ; \xi \wedge \xi\right\rangle
$$

The function $f$ is clearly ext. polyconvex but not convex.

(ii) The study of the implications and counter implications for ext. one convexity, ext. quasiconvexity and ext. polyconvexity is therefore complete, except for the last implication, namely

$$
\text { f ext. quasiconvex } \underset{\nLeftarrow}{\Rightarrow} \text { ext. one convex, }
$$

only for the case $k=n-2 \geq 2$ even (including $k=2$ and $n=4$ ), which remains open.

(iii) It is interesting to read the theorem when $k=2$.

- If $n=2$ or $n=3$, then

$$
f \text { convex } \Leftrightarrow f \text { ext. polyconvex } \Leftrightarrow f \text { ext. quasiconvex } \Leftrightarrow f \text { ext. one convex. }
$$

- If $n \geq 4$, then

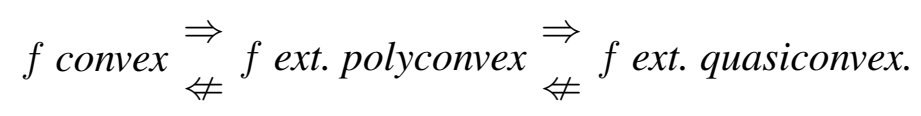

- If $n \geq 5$, then

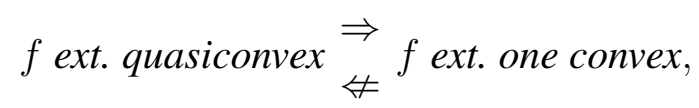

while the case $n=4$ remains open. 
Proof. (i) Step 1. The implication

$$
f \text { convex } \Rightarrow f \text { ext. polyconvex }
$$

is trivial.

Step 2. The statement

$$
f \text { ext. polyconvex } \Rightarrow f \text { ext. quasiconvex }
$$

is proved as follows. Observe first that if $\xi \in \Lambda^{k}$ and $\omega \in W_{0}^{1, \infty}\left(\Omega ; \Lambda^{k-1}\right)$, then

$$
\int_{\Omega}(\xi+d \omega)^{s}=\xi^{s} \text { meas } \Omega, \quad \text { for every integer } s .
$$

We proceed by induction on $s$. The case $s=1$ is trivial, so we assume that the result has already been established for $s-1$ and we prove it for $s$. Note that

$$
\begin{aligned}
(\xi+d \omega)^{s} & =\xi \wedge(\xi+d \omega)^{s-1}+d \omega \wedge(\xi+d \omega)^{s-1} \\
& =\xi \wedge(\xi+d \omega)^{s-1}+d\left[\omega \wedge(\xi+d \omega)^{s-1}\right] .
\end{aligned}
$$

Integrating, using induction for the first integral and the fact that $\omega=0$ on $\partial \Omega$ for the second one, we have indeed shown (1). We can now conclude. Since $f$ is ext. polyconvex, we can find a convex function

$$
F: \Lambda^{k} \times \Lambda^{2 k} \times \cdots \times \Lambda^{[n / k] k} \rightarrow \mathbb{R}
$$

such that

$$
f(\xi)=F\left(\xi, \xi^{2}, \ldots, \xi^{[n / k]}\right) .
$$

Using Jensen inequality we find,

$$
\frac{1}{\operatorname{meas} \Omega} \int_{\Omega} f(\xi+d \omega) \geq F\left(\frac{1}{\operatorname{meas} \Omega} \int_{\Omega}(\xi+d \omega), \ldots, \frac{1}{\operatorname{meas} \Omega} \int_{\Omega}(\xi+d \omega)^{[n / k]}\right) \text {. }
$$

Invoking (1), we have indeed obtained that

$$
\int_{\Omega} f(\xi+d \omega) \geq f(\xi) \text { meas } \Omega
$$

and the proof of Step 2 is complete.

Step 3. It follows from Lemma 2.7 that

$$
f \text { ext. quasiconvex } \Rightarrow f \text { ext. one convex. }
$$

With Lemma2.7 at our disposal, the proof is very similar to that of the case of the gradient (cf. Theorem 5.3 in [8]) and is omitted. See [17] for the details. This concludes the proof of (i). 
(ii) In all the cases under consideration any $\xi \in \Lambda^{k}$ is 1-divisible (cf. Proposition 2.43 in [5]). Hence, the result

$$
f \text { convex } \Leftrightarrow f \text { ext. polyconvex } \Leftrightarrow f \text { ext. quasiconvex } \Leftrightarrow f \text { ext. one convex }
$$

then follows at once. The extra statement (i.e. when $k$ is odd or $2 k>n$ )

$$
f \text { convex } \Leftrightarrow f \text { ext. polyconvex. }
$$

is proved in Remark 2.2 (ii) and Proposition 2.14.

(iii) The statement that

$$
f \text { ext. polyconvex } \underset{\nLeftarrow}{\Rightarrow} f \text { ext. quasiconvex, }
$$

when $3 \leq k \leq n-3$ or $k=n-2 \geq 4$ is even follows from Theorem 4.5 (v) and from Proposition 4.11 when $k=2$ and $n \geq 4$ (for $k=2$ and $n \geq 6$, we can also apply Theorem 4.5 (ii)).

The statement that if $2 \leq k \leq n-3$ (and thus $n \geq k+3 \geq 5$ ), then

$$
f \text { ext. quasiconvex } \underset{\nLeftarrow}{\Rightarrow} f \text { ext. one convex, }
$$

follows from Theorem 4.8 ,

We also have the following elementary properties.

Proposition 2.10. Let $1 \leq k \leq n$ and $f: \Lambda^{k} \rightarrow \mathbb{R}$.

(i) Any ext. one convex function is locally Lipschitz.

(ii) If $f$ is ext. one convex and $C^{2}$, for every $\xi \in \Lambda^{k}, \alpha \in \Lambda^{k-1}$ and $\beta \in \Lambda^{1}$,

$$
\sum_{I, J \in \mathcal{T}_{k}^{n}} \frac{\partial^{2} f(\xi)}{\partial \xi_{I} \partial \xi_{J}}(\alpha \wedge \beta)_{I}(\alpha \wedge \beta)_{J} \geq 0 .
$$

Proof. (i) The fact that $f$ is locally Lipschitz follows from the observation that any ext. one convex function is in fact separately convex. These last functions are known to be locally Lipschitz (cf. Theorem 2.31 in [8]).

(ii) We next assume that $f$ is $C^{2}$. By definition the function

$$
g: t \rightarrow g(t)=f(\xi+t \alpha \wedge \beta)
$$

is convex for every $\xi \in \Lambda^{k}, \alpha \in \Lambda^{k-1}$ and $\beta \in \Lambda^{1}$. Since $f$ is $C^{2}$, our claim follows from the fact that $g^{\prime \prime}(0) \geq 0$.

We now give an equivalent formulation of ext. quasiconvexity, but before that we need the following notation. 
Notation 2.11. Let $\Omega \subset \mathbb{R}^{n}$ be a smooth, open set. We define

$$
W_{\delta, T}^{1, \infty}\left(\Omega ; \Lambda^{k}\right)=\left\{\omega \in W^{1, \infty}\left(\Omega ; \Lambda^{k}\right): \delta \omega=0 \text { in } \Omega \text { and } \nu \wedge \omega=0 \text { on } \partial \Omega\right\},
$$

where $\nu$ is the outward unit normal to $\partial \Omega$.

Proposition 2.12. Let $f: \Lambda^{k} \rightarrow \mathbb{R}$ be continuous. The following statements are equivalent.

(i) $f$ is ext. quasiconvex.

(ii) For every bounded smooth open set $\Omega \subset \mathbb{R}^{n}, \psi \in W_{\delta, T}^{1, \infty}\left(\Omega ; \Lambda^{k-1}\right)$ and $\xi \in \Lambda^{k}$,

$$
\int_{\Omega} f(\xi+d \psi) \geq f(\xi) \text { meas } \Omega \text {. }
$$

Remark 2.13. Given a function $f: \Lambda^{k} \rightarrow \mathbb{R}$, the ext. quasiconvex envelope, which is the largest ext quasiconvex function below $f$, is given by (as in Theorem 6.9 of [8])

$$
\begin{aligned}
Q_{\text {ext }} f(\xi) & =\inf \left\{\frac{1}{\operatorname{meas} \Omega} \int_{\Omega} f(\xi+d \omega): \omega \in W_{0}^{1, \infty}\left(\Omega ; \Lambda^{k-1}\right)\right\} \\
& =\inf \left\{\frac{1}{\operatorname{meas} \Omega} \int_{\Omega} f(\xi+d \psi): \psi \in W_{\delta, T}^{1, \infty}\left(\Omega ; \Lambda^{k-1}\right)\right\} .
\end{aligned}
$$

Proof. (ii) $\Rightarrow$ (i): Let $\Omega \subset \mathbb{R}^{n}$ be a bounded smooth open set, $\xi \in \Lambda^{k}$ and let $\omega \in$ $W_{0}^{1, \infty}\left(\Omega ; \Lambda^{k-1}\right)$. Using density, we find $\omega_{\epsilon} \in C_{0}^{\infty}\left(\Omega ; \Lambda^{k-1}\right)$ such that

$$
\sup _{\epsilon>0}\left\|\nabla \omega_{\epsilon}\right\|_{L^{\infty}}<\infty \text { and } \omega_{\epsilon} \rightarrow \omega, \text { in } W^{1,2}\left(\Omega ; \Lambda^{k-1}\right) \text {. }
$$

Appealing to Theorem 7.2 in [5], we now find $\psi_{\epsilon} \in C_{\delta, T}^{\infty}\left(\Omega ; \Lambda^{k-1}\right)$ such that

$$
\left\{\begin{array}{cl}
d \psi_{\epsilon}=d \omega_{\epsilon} & \text { in } \Omega \\
\delta \psi_{\epsilon}=0 & \text { in } \Omega \\
\nu \wedge \psi_{\epsilon}=0 & \text { on } \partial \Omega .
\end{array}\right.
$$

We use (2) to apply dominated convergence theorem to obtain

$$
\int_{\Omega} f(\xi+d \omega)=\lim _{\epsilon \rightarrow 0} \int_{\Omega} f\left(\xi+d \omega_{\epsilon}\right)=\lim _{\epsilon \rightarrow 0} \int_{\Omega} f\left(\xi+d \psi_{\epsilon}\right) \geq f(\xi) \text { meas } \Omega,
$$

where we have used (ii) in the last step. Therefore, $f$ is ext. quasiconvex.

(i) $\Rightarrow$ (ii): Let $\psi \in W_{\delta, T}^{1, \infty}\left(\Omega ; \Lambda^{k-1}\right)$. Then, by Theorem 8.16 in [5], we can find $\omega \in W_{0}^{1,2}\left(\Omega ; \Lambda^{k-1}\right)$ such that

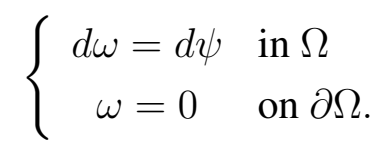

With a similar argument as above, we infer that

$$
\int_{\Omega} f(\xi+d \psi)=\int_{\Omega} f(\xi+d \omega)=\lim _{\epsilon \rightarrow 0} \int_{\Omega} f\left(\xi+d \omega_{\epsilon}\right) \geq f(\xi) \text { meas } \Omega,
$$

as claimed. 
We finally have also another formulation of ext. polyconvexity.

Proposition 2.14. Let $f: \Lambda^{k} \rightarrow \mathbb{R}$. The following statements are then equivalent.

(i) The function $f$ is ext. polyconvex.

(ii) For every $\xi \in \Lambda^{k}$, there exist $c_{s}=c_{s}(\xi) \in \Lambda^{k s}, 1 \leq s \leq[n / k]$, such that

$$
f(\eta) \geq f(\xi)+\sum_{s=1}^{[n / k]}\left\langle c_{s}(\xi) ; \eta^{s}-\xi^{s}\right\rangle, \quad \text { for every } \eta \in \Lambda^{k}
$$

(iii) Let

$$
N=\sum_{s=1}^{[n / k]}\left(\begin{array}{l}
n \\
s k
\end{array}\right)
$$

For every $t_{i} \geq 0$ with $\sum_{i=1}^{N+1} t_{i}=1$ and every $\xi_{i} \in \Lambda^{k}$ such that

$$
\sum_{i=1}^{N+1} t_{i} \xi_{i}^{s}=\left(\sum_{i=1}^{N+1} t_{i} \xi_{i}\right)^{s}, \quad \text { for every } 1 \leq s \leq[n / k]
$$

the following inequality holds

$$
f\left(\sum_{i=1}^{N+1} t_{i} \xi_{i}\right) \leq \sum_{i=1}^{N+1} t_{i} f\left(\xi_{i}\right)
$$

Proof. (i) $\Rightarrow$ (ii): Since $f$ is ext. polyconvex, there exists a convex function $F$ such that

$$
f(\xi)=F\left(\xi, \xi^{2}, \ldots, \xi^{[n / k]}\right)
$$

$F$ being convex, for every $\xi \in \Lambda^{k}$, there exists $c_{s}=c_{s}(\xi) \in \Lambda^{k s}, 1 \leq s \leq[n / k]$, such that, for all $\eta \in \Lambda^{k}$,

$$
f(\eta)-f(\xi)=F\left(\eta, \ldots, \eta^{[n / k]}\right)-F\left(\xi, \ldots, \xi^{[n / k]}\right) \geq \sum_{s=1}^{[n / k]}\left\langle c_{s} ; \eta^{s}-\xi^{s}\right\rangle
$$

as claimed.

(ii) $\Rightarrow$ (i): Conversely assume that the inequality is valid and, for $\theta=\left(\theta_{1}, \ldots, \theta_{[n / k]}\right) \in$ $\Lambda^{k} \times \cdots \times \Lambda^{[n / k] k}$, we define

$$
F(\theta)=\sup _{\xi \in \Lambda^{k}}\left\{f(\xi)+\sum_{s=1}^{[n / k]}\left\langle c_{s}(\xi) ; \theta_{s}-\xi^{s}\right\rangle\right\}
$$

Clearly $F$ is convex as a supremum of affine functions. Moreover if

$$
\theta=\left(\eta, \ldots, \eta^{[n / k]}\right)
$$


then, in view of the inequality, the supremum is attained by $\xi=\eta$, i.e.

$$
f(\eta)=F\left(\eta, \ldots, \eta^{[n / k]}\right)
$$

and thus $f$ is ext. polyconvex.

(i) $\Rightarrow$ (iii): Since $f$ is ext. polyconvex, there exists a convex function $F$ such that

$$
f(\xi)=F\left(\xi, \xi^{2}, \ldots, \xi^{[n / k]}\right) .
$$

The convexity of $F$ implies that

$$
\begin{aligned}
f\left(\sum_{i=1}^{N+1} t_{i} \xi_{i}\right) & =F\left(\left(\sum_{i=1}^{N+1} t_{i} \xi_{i}\right), \ldots,\left(\sum_{i=1}^{N+1} t_{i} \xi_{i}\right)^{[n / k]}\right) \\
& =F\left(\sum_{i=1}^{N+1} t_{i}\left(\xi_{i}, \ldots, \xi_{i}^{[n / k]}\right)\right) \leq \sum_{i=1}^{N+1} t_{i} F\left(\xi_{i}, \ldots, \xi_{i}^{[n / k]}\right) \\
& =\sum_{i=1}^{N+1} t_{i} f\left(\xi_{i}\right) .
\end{aligned}
$$

(iii) $\Rightarrow$ (i): The proof is based on Carathéodory theorem and follows exactly as in Theorem 5.6 in [8].

\section{The quasiaffine case}

\subsection{Some preliminary results}

We start with two elementary results.

Lemma 3.1. Let $f: \Lambda^{k} \rightarrow \mathbb{R}$ be ext. one affine with $1 \leq k \leq n$. Then

$$
f\left(\xi+\sum_{i=1}^{N} t_{i} \alpha_{i} \wedge a\right)=f(\xi)+\sum_{i=1}^{N} t_{i}\left[f\left(\xi+\alpha_{i} \wedge a\right)-f(\xi)\right]
$$

for every $t_{i} \in \mathbb{R}, \xi \in \Lambda^{k}, \alpha_{i} \in \Lambda^{k-1}, a \in \Lambda^{1}$.

Proof. Step 1. Since $f$ is ext. one affine,

$$
f(\xi+t \alpha \wedge a)=f(\xi)+t[f(\xi+\alpha \wedge a)-f(\xi)]
$$

Step 2. Let us first prove that

$$
f(\xi+\alpha \wedge a+\beta \wedge a)+f(\xi)=f(\xi+\alpha \wedge a)+f(\xi+\beta \wedge a) .
$$


First assume that $s \neq 0$. We have, using Step 1, that

$$
\begin{aligned}
f(\xi+s \alpha \wedge a+\beta \wedge a) & =f\left(\xi+s\left(\alpha+\frac{1}{s} \beta\right) \wedge a\right) \\
& =f(\xi)+s\left[f\left(\xi+\left(\alpha+\frac{1}{s} \beta\right) \wedge a\right)-f(\xi)\right]
\end{aligned}
$$

and hence, using Step 1 again,

$$
\begin{aligned}
& f(\xi+s \alpha \wedge a+\beta \wedge a) \\
& =f(\xi)+s\left\{f(\xi+\alpha \wedge a)+\frac{1}{s}[f(\xi+\alpha \wedge a+\beta \wedge a)-f(\xi+\alpha \wedge a)]-f(\xi)\right\} \\
& =f(\xi)+s[f(\xi+\alpha \wedge a)-f(\xi)]+[f(\xi+\alpha \wedge a+\beta \wedge a)-f(\xi+\alpha \wedge a)] .
\end{aligned}
$$

Since $f$ is continuous, we have the result by letting $s \rightarrow 0$.

Step 3. We now prove the claim. We proceed by induction. The case $N=1$ is just Step 1 . We first use the induction hypothesis to write

$$
\begin{aligned}
& f\left(\xi+\sum_{i=1}^{N} t_{i} \alpha_{i} \wedge a\right)=f\left(\xi+t_{N} \alpha_{N} \wedge a+\sum_{i=1}^{N-1} t_{i} \alpha_{i} \wedge a\right) \\
& =f\left(\xi+t_{N} \alpha_{N} \wedge a\right)+\sum_{i=1}^{N-1} t_{i}\left[f\left(\xi+t_{N} \alpha_{N} \wedge a+\alpha_{i} \wedge a\right)-f\left(\xi+t_{N} \alpha_{N} \wedge a\right)\right] .
\end{aligned}
$$

We then appeal to Step 1 to get

$$
\begin{aligned}
& f\left(\xi+\sum_{i=1}^{N} t_{i} \alpha_{i} \wedge a\right)=f(\xi)+t_{N}\left[f\left(\xi+\alpha_{N} \wedge a\right)-f(\xi)\right] \\
& +\sum_{i=1}^{N-1} t_{i}\left\{\begin{array}{c}
f\left(\xi+\alpha_{i} \wedge a\right)+t_{N}\left[f\left(\xi+\alpha_{i} \wedge a+\alpha_{N} \wedge a\right)-f\left(\xi+\alpha_{i} \wedge a\right)\right] \\
-f(\xi)-t_{N}\left[f\left(\xi+\alpha_{N} \wedge a\right)-f(\xi)\right]
\end{array}\right\},
\end{aligned}
$$

and thus

$$
\begin{aligned}
& f\left(\xi+\sum_{i=1}^{N} t_{i} \alpha_{i} \wedge a\right)=f(\xi)+\sum_{i=1}^{N} t_{i}\left[f\left(\xi+\alpha_{i} \wedge a\right)-f(\xi)\right] \\
& +t_{N} \sum_{i=1}^{N-1} t_{i}\left\{f\left(\xi+\alpha_{i} \wedge a+\alpha_{N} \wedge a\right)-f\left(\xi+\alpha_{i} \wedge a\right)-f\left(\xi+\alpha_{N} \wedge a\right)+f(\xi)\right\} .
\end{aligned}
$$

Appealing to Step 2, we see that the last term vanishes and therefore the induction reasoning is complete and this achieves the proof of the lemma.

We have as an immediate consequence the following result. 
Corollary 3.2. Let $f: \Lambda^{k} \rightarrow \mathbb{R}$ be ext. one affine with $1 \leq k \leq n$. Then

$$
\begin{aligned}
& {[f(\xi+\alpha \wedge a+\beta \wedge b)-f(\xi)]+[f(\xi+\beta \wedge a+\alpha \wedge b)-f(\xi)]} \\
& =[f(\xi+\alpha \wedge a)-f(\xi)]+[f(\xi+\beta \wedge a)-f(\xi)] \\
& +[f(\xi+\alpha \wedge b)-f(\xi)]+[f(\xi+\beta \wedge b)-f(\xi)]
\end{aligned}
$$

for every $\xi \in \Lambda^{k}, \alpha, \beta \in \Lambda^{k-1}, a, b \in \Lambda^{1}$.

\subsection{The main theorem}

Theorem 3.3. Let $1 \leq k \leq n$ and $f: \Lambda^{k} \rightarrow \mathbb{R}$. The following statements are then equivalent.

(i) $f$ is ext. polyaffine.

(ii) $f$ is ext. quasiaffine.

(iii) $f$ is ext. one affine.

(iv) For every $0 \leq s \leq[n / k]$, there exist $c_{s} \in \Lambda^{k s}$ such that,

$$
f(\xi)=\sum_{s=0}^{[n / k]}\left\langle c_{s} ; \xi^{s}\right\rangle, \text { for every } \xi \in \Lambda^{k}
$$

Remark 3.4. When $k$ is odd (since then $\xi^{s}=0$ for every $s \geq 2$ ) or when $2 k>n$ (in particular when $k=n$ or $k=n-1$ ), then all the statements are equivalent to $f$ being affine.

Proof. The statements

$$
(i) \Rightarrow(i i) \Rightarrow(i i i)
$$

follow at once from Theorem 2.8 . The statement

$$
(i v) \Rightarrow(i)
$$

is a direct consequence of the definition of ext. polyconvexity. So it only remains to prove

$$
(i i i) \Rightarrow(i v)
$$

We divide the proof into three steps.

Step 1. We first prove that $f$ is a polynomial of degree at most $n$ and is of the form

$$
f(\xi)=\sum_{s=0}^{n} f_{s}(\xi)
$$


where, for each $s=0, \ldots, n, f_{s}$ is a homogeneous polynomial of degree $s$ and is ext. one affine. To prove (3), let us proceed by induction on the dimension $n$. The case $n=1$ is trivial to check. For each $\xi \in \Lambda^{k}$, we write

$$
\xi=\sum_{I \in \mathcal{T}_{k}^{n}, 1 \in I} \xi_{I} e^{I}+\xi_{N}, \text { where } \xi_{N}=\sum_{I \in \mathcal{T}_{k}^{n}, 1 \notin I} \xi_{I} e^{I} .
$$

Note that, $\xi_{N} \in \Lambda^{k}\left(\left\{e^{1}\right\}^{\perp}\right)$. Invoking Lemma 3.1, we obtain that

$$
f(\xi)=f\left(\xi_{N}\right)+\sum_{I \in \mathcal{T}_{k}^{n}, 1 \in I} \xi_{I}\left[f\left(\xi_{N}+e^{I}\right)-f\left(\xi_{N}\right)\right] .
$$

Since $f$ is ext. one affine on $\Lambda^{k}\left(\left\{e^{1}\right\}^{\perp}\right)$, applying the induction hypothesis to $\left.f\right|_{\Lambda^{k}}\left(\left\{e^{1}\right\}^{\perp}\right)$ and $\left.f\left(e^{I}+\cdot\right)\right|_{\Lambda^{k}\left(\left\{e^{1}\right\}^{\perp}\right)}$, we deduce that both are polynomials of degree at most $(n-1)$. Hence, $f$ is a polynomial of degree at most $n$. This proves the claim by induction. That each of $f_{s}$ is ext. one affine, follows from the fact that each $f_{s}$ has a different degree of homogeneity.

Step 2. We now show that $f$ is, in fact, a polynomial of degree at most $[n / k]$, which is equivalent to proving that each $f_{s}$ in (3) is a polynomial of degree at most $[n / k]$. Since $f_{s}$ is a homogeneous polynomial of degree $s$, we can write

$$
f_{s}(\xi)=\sum_{I^{1}, \ldots, I^{s} \in \mathcal{T}_{k}^{n}} d_{I^{1} \ldots I^{s}} \xi_{I^{1}} \cdots \xi_{I^{s}}
$$

where $d_{I^{1 \ldots I^{s}}} \in \mathbb{R}$. It is enough to prove that, for some $I^{1}, \ldots, I^{s} \in \mathcal{T}_{k}^{n}$, whenever $d_{I^{1 \ldots I^{s}}} \neq 0$, we have,

$$
I^{p} \cap I^{q}=\emptyset, \text { for all } p, q=1, \ldots, s ; p \neq q .
$$

Let us suppose to the contrary that, for some $p, q, p \neq q$, we have $I^{p} \cap I^{q} \neq \emptyset$. For $t \in \mathbb{R}$, let us define

$$
\xi(t)=t\left(e^{I^{p}}+e^{I^{q}}\right)+\sum_{\substack{a=1 \\ a \neq p, q}}^{s} e^{I^{a}}
$$

We, therefore, have according to (4) that, for all $t \in \mathbb{R}$,

$$
f_{s}(\xi(t))=t^{2} d_{I^{1} \cdots I^{s}}, \text { for all } t \in \mathbb{R} .
$$

On the other hand, using Lemma 3.1, it follows that

$$
f_{s}(\xi(t))=f_{s}\left(\sum_{\substack{a=1 \\ a \neq p, q}}^{s} e^{I^{a}}+t\left(e^{I^{p}}+e^{I^{q}}\right)\right)=f_{s}(\xi(0))+t\left[f_{s}(\xi(1))-f_{s}(\xi(0))\right]
$$


which is an affine function of $t$. This proves the claim by contradiction.

Step 3. Henceforth, to avoid any ambiguity, let us fix an order in which multiindices $I^{1}, \cdots, I^{s}$ are considered to appear in Equation (4) and we choose the order to that

$$
i_{1}^{1}<\cdots<i_{1}^{s}
$$

where $i_{1}^{j}$ is the first element of $I^{j}$, for all $j=1, \ldots, s$. With the aforementioned order in force, we re-arrange Equation (4) to have

$$
f(\xi)=\sum_{r=0}^{[n / k]} f_{s}(\xi), \quad \text { where } \quad f_{s}(\xi)=\sum_{I^{1}, \ldots, I^{s}} c_{I^{1} \cdots I^{s}} \xi_{I^{1}} \cdots \xi_{I^{s}}
$$

with $c_{I^{1} \ldots I^{s}} \in \mathbb{R} \backslash\{0\}$, and the ordered multiindices $I^{1}, \ldots, I^{s} \in \mathcal{T}_{k}^{n}$ with $i_{1}^{1}<\cdots<i_{1}^{s}$. Note that, the theorem is proved once we show that

$$
f_{s}(\xi)=\left\langle c_{s} ; \xi^{s}\right\rangle
$$

which is equivalent to proving that,

$$
c_{J^{1} \ldots J^{s}}=\operatorname{sgn}(\sigma) c_{I^{1} \ldots I^{s}}
$$

for all $I^{1}, \ldots, I^{s}, J^{1}, \ldots, J^{s} \in \mathcal{T}_{k}^{n}$ satisfying $J^{1} \cup \cdots \cup J^{s}=I^{1} \cup \cdots \cup I^{s}, \sigma\left(J^{1} J^{2} \cdots J^{s}\right)=$ $\left(I^{1} I^{2} \cdots I^{s}\right)$, where $\sigma \in \mathcal{S}^{s k}$ is the permutation of indices that respects the aforementioned order.

Step 3.1. Before concluding the proof, we observe that, for all $s=2, \ldots, n$,

$$
f_{s}\left(\sum_{i=1}^{s-1} t_{i} \alpha_{i}\right)=0
$$

where $t_{i} \in \mathbb{R}$ and $\alpha_{i}$ is an element of the standard basis of $\Lambda^{k}$. This is a direct consequence of the fact that $f_{s}$ is homogeneous of degree $s$ and that

$$
\xi=\sum_{i=1}^{s-1} t_{i} \alpha_{i}
$$

has at most $(s-1)$ coefficients that are non-zero.

Step 3.2. We finally establish Equation (6). Let $I^{1}, \ldots, I^{s}, J^{1}, \ldots, J^{s} \in \mathcal{T}_{k}^{n}$ satisfy

$$
J^{1} \cup \cdots \cup J^{s}=I^{1} \cup \cdots \cup I^{s} \text {, and } \sigma\left(J^{1} J^{2} \cdots J^{s}\right)=\left(I^{1} I^{2} \cdots I^{s}\right),
$$

where $\sigma \in \mathcal{S}^{s k}$ is the permutation of indices that respects the order. We claim that

$$
c_{J^{1} \ldots J^{s}}=\operatorname{sgn}(\sigma) c_{I^{1 \ldots I^{s}}} .
$$


Since any permutation that respects the ordering scheme is a product (unique up to parity) of transpositions each of which respects the ordering, it is enough to prove the result for the case where $\sigma$ is a transposition that respects the ordering. Hence, Equation (7) reduces to proving that

$$
c_{I^{1 \ldots I^{s}}}=-c_{J^{1} \ldots J^{s}} .
$$

Let us write

$$
I^{1}=\left(i_{1}^{1}, \ldots, i_{k}^{1}\right), \ldots, I^{s}=\left(i_{1}^{s}, \ldots, i_{k}^{s}\right),
$$

and

$$
J^{1}=\left(j_{1}^{1}, \ldots, j_{k}^{1}\right), \ldots, J^{s}=\left(j_{1}^{s}, \ldots, j_{k}^{s}\right) .
$$

Then, $i_{1}^{1}<\cdots<i_{1}^{s} \quad$ and $\quad j_{1}^{1}<\cdots<j_{1}^{s}$. Since $\sigma$ respects the ordering scheme, $\sigma$ flips two indices $i_{r_{1}}^{q_{1}}$ and $i_{r_{2}}^{q_{2}}$, with $q_{1} \neq q_{2}$ and leaves other indices fixed. Note that, from (5), we have

$$
c_{I^{1} \ldots I^{s}}=f_{s}\left(\sum_{m=1}^{s} e^{I^{m}}\right)=f_{s}\left(\sum_{m=1}^{s} e^{i_{1}^{m}} \wedge \cdots \wedge e^{i_{k}^{m}}\right),
$$

and

$$
c_{J^{1} \ldots J^{s}}=f_{s}\left(\sum_{m=1}^{s} e^{J^{m}}\right)=f_{s}\left(\sum_{m=1}^{s} e^{j_{1}^{m}} \wedge \cdots \wedge e^{j_{k}^{m}}\right) .
$$

Since $f_{s}$ is ext. one affine, setting

$$
\begin{gathered}
a=e^{i_{r_{1}}^{q_{1}}}, \quad b=e^{i_{r_{2}}}, \quad \xi=\sum_{\substack{m=1 \\
m \neq q_{1}, q_{2}}}^{s} e^{i_{1}^{m}} \wedge \cdots \wedge e^{i_{k}^{m}}=\sum_{\substack{m=1 \\
m \neq q_{1}, q_{2}}}^{s} e^{I^{m}}, \\
\alpha= \pm e^{i_{1}^{q_{1}}} \wedge \cdots \wedge \widehat{e}^{\widehat{q_{1}}} \wedge \cdots \wedge e^{i_{k}^{q_{1}}} \quad \text { and } \quad \beta= \pm e^{i_{1}^{q_{2}}} \wedge \cdots \wedge e^{\widehat{q_{2}}} \wedge \cdots \wedge e^{i_{k}^{q_{2}}},
\end{gathered}
$$

with signs being chosen appropriately so that

$$
\alpha \wedge a=e^{I^{q_{1}}}=e^{i_{1}^{q_{1}}} \wedge \cdots \wedge e^{i_{k}^{q_{1}}} \quad \text { and } \quad \beta \wedge b=e^{I^{q_{2}}}=e^{i_{1}^{q_{2}}} \wedge \cdots \wedge e^{i_{k}^{q_{2}}},
$$

we can apply Corollary 3.2 to $f_{s}$ to obtain

$$
\begin{aligned}
& {\left[f_{s}(\xi+\alpha \wedge a+\beta \wedge b)-f_{s}(\xi)\right]+\left[f_{s}(\xi+\beta \wedge a+\alpha \wedge b)-f_{s}(\xi)\right]} \\
& =\left[f_{s}(\xi+\alpha \wedge a)-f_{s}(\xi)\right]+\left[f_{s}(\xi+\beta \wedge b)-f_{s}(\xi)\right] \\
& +\left[f_{s}(\xi+\beta \wedge a)-f_{s}(\xi)\right]+\left[f_{s}(\xi+\alpha \wedge b)-f_{s}(\xi)\right] .
\end{aligned}
$$

Using Step 3.1, all except $f_{s}(\xi+\alpha \wedge a+\beta \wedge b)$ and $f_{s}(\xi+\beta \wedge a+\alpha \wedge b)$ are 0. Hence, we deduce

$$
f_{s}(\xi+\alpha \wedge a+\beta \wedge b)=-f_{s}(\xi+\beta \wedge a+\alpha \wedge b),
$$

which, together with (9) and (10) proves (8). This concludes the proof of Step 3.2 and thus of the theorem. 


\section{Some examples}

\subsection{The quadratic case}

\subsubsection{Some preliminary results}

Before stating the main theorem on quadratic forms, we need a lemma whose proof is straightforward.

Lemma 4.1. Let $1 \leq k \leq n, M: \Lambda^{k} \rightarrow \Lambda^{k}$ be a symmetric linear operator and $f: \Lambda^{k} \rightarrow$ $\mathbb{R}$ be such that, for every $\xi \in \Lambda^{k}$,

$$
f(\xi)=\langle M \xi ; \xi\rangle .
$$

The following statements then hold true.

(i) $f$ is ext. polyconvex if and only if there exists $\beta \in \Lambda^{2 k}$ so that,

$$
f(\xi) \geq\langle\beta ; \xi \wedge \xi\rangle, \text { for every } \xi \in \Lambda^{k} .
$$

(ii) $f$ is ext. quasiconvex if and only if

$$
\int_{\Omega} f(d \omega) \geq 0
$$

for every bounded open set $\Omega \subset \mathbb{R}^{n}$ and $\omega \in W_{0}^{1, \infty}\left(\Omega ; \Lambda^{k-1}\right)$.

(iii) $f$ is ext. one convex if and only if, for every $a \in \Lambda^{k-1}$ and $b \in \Lambda^{1}$,

$$
f(a \wedge b) \geq 0,
$$

\subsubsection{Some examples}

We start with the following example that will be used in Theorem 4.5 below.

Proposition 4.2. Let $2 \leq k \leq n-2$. Let $\alpha \in \Lambda^{k}$ be not 1-divisible, then there exists $c>0$ such that

$$
f(\xi)=|\xi|^{2}-c(\langle\alpha ; \xi\rangle)^{2},
$$

is ext. quasiconvex but not convex. If, in addition $\alpha \wedge \alpha=0$, then the above $f$, for an appropriate $c$, is ext. quasiconvex but not ext. polyconvex.

Remark 4.3. (i) It is easy to see that $\alpha$ is not 1-divisible if and only if

$$
\operatorname{rank}[* \alpha]=n .
$$

This results from Remark 2.44 (iv) (with the help of Proposition 2.33 (iii)) in [5]. Such an $\alpha$ always exists if either of the following holds (see Propositions 2.37 (ii) and 2.43 in [5]) 
- $k=n-2 \geq 2$ is even,

$-2 \leq k \leq n-3$.

For example,

$$
\alpha=e^{1} \wedge e^{2} \wedge e^{3}+e^{4} \wedge e^{5} \wedge e^{6} \in \Lambda^{3}\left(\mathbb{R}^{6}\right),
$$

is not 1-divisible.

(ii) Note that, when $k=2$ every form $\alpha$ such that $\alpha \wedge \alpha=0$ is necessarily 1-divisible. While, as soon as $k$ is even and $4 \leq k \leq n-2$, there exists $\alpha$ that is not 1 -divisible, but $\alpha \wedge \alpha=0$. For example, when $k=4$,

$$
\alpha=e^{1} \wedge e^{2} \wedge e^{3} \wedge e^{4}+e^{1} \wedge e^{2} \wedge e^{5} \wedge e^{6}+e^{3} \wedge e^{4} \wedge e^{5} \wedge e^{6} \in \Lambda^{4}\left(\mathbb{R}^{6}\right)
$$

Proof. Since the function is quadratic, the notions of ext. one convexity and ext. quasiconvexity are equivalent (see Theorem 4.5). We therefore only need to discuss the ext. one convexity. We divide the proof into two steps.

Step 1. We first show that if

$$
\frac{1}{c}=\sup \left\{(\langle\alpha ; a \wedge b\rangle)^{2}: a \in \Lambda^{k-1}, b \in \Lambda^{1},|a \wedge b|=1\right\}
$$

then

$$
\frac{1}{c}<|\alpha|^{2}
$$

We prove this statement as follows. Let $a_{s} \in \Lambda^{k-1}, b_{s} \in \Lambda^{1}$ be a maximizing sequence. Up to a subsequence that we do not relabel, we find that there exists $\lambda \in \Lambda^{k}$ so that

$$
a_{s} \wedge b_{s} \rightarrow \lambda \text { with } \quad|\lambda|=1
$$

Similarly, up to a subsequence that we do not relabel, we have that there exists $\bar{b} \in \Lambda^{1}$ so that

$$
\frac{b_{s}}{\left|b_{s}\right|} \rightarrow \bar{b}
$$

Since

$$
a_{s} \wedge b_{s} \wedge \frac{b_{s}}{\left|b_{s}\right|}=0
$$

we deduce that

$$
\lambda \wedge \bar{b}=0 .
$$

Appealing to Cartan lemma (see Theorem 2.42 in [5]), we find that there exists $\bar{a} \in \Lambda^{k-1}$ such that

$$
\lambda=\bar{a} \wedge \bar{b} \quad \text { with } \quad|\bar{a} \wedge \bar{b}|=1 .
$$

We therefore have found that

$$
\frac{1}{c}=(\langle\alpha ; \bar{a} \wedge \bar{b}\rangle)^{2}
$$


Note that $\frac{1}{c}<|\alpha|^{2}$, otherwise $\bar{a} \wedge \bar{b}$ would be parallel to $\alpha$ and thus $\alpha$ would be 1 -divisible which contradicts the hypothesis.

Step 2. So let

$$
f(\xi)=|\xi|^{2}-c(\langle\alpha ; \xi\rangle)^{2}
$$

(i) Observe that $f$ is not convex since $c|\alpha|^{2}>1$ (by Step 1). Indeed,

$$
f\left(\frac{1}{2} \alpha+\frac{1}{2}(-\alpha)\right)=f(0)=0>|\alpha|^{2}\left(1-c|\alpha|^{2}\right)=f(\alpha)=\frac{1}{2} f(\alpha)+\frac{1}{2} f(-\alpha) .
$$

(ii) However, $f$ is ext. one convex (and thus, invoking Theorem 4.5, $f$ is ext. quasiconvex). Indeed, let

$$
g(t)=f(\xi+t a \wedge b)=|\xi+t a \wedge b|^{2}-c(\langle\alpha ; \xi+t a \wedge b\rangle)^{2}
$$

Note that

$$
g^{\prime \prime}(t)=2\left[|a \wedge b|^{2}-c(\langle\alpha ; a \wedge b\rangle)^{2}\right],
$$

which is non-negative by Step 1. Thus $g$ is convex.

(iii) Let $\alpha \wedge \alpha=0$ and assume, for the sake of contradiction, that $f$ is ext. polyconvex. Then there should exist (cf. Lemma 4.1) $\beta \in \Lambda^{2 k}$ so that, for every $\xi \in \Lambda^{k}$,

$$
f(\xi) \geq\langle\beta ; \xi \wedge \xi\rangle
$$

This is clearly impossible, in view of the fact that $c|\alpha|^{2}>1$, since choosing $\xi=\alpha$, we get

$$
f(\alpha)=|\alpha|^{2}\left(1-c|\alpha|^{2}\right)<0=\langle\beta ; \alpha \wedge \alpha\rangle .
$$

The proof is therefore complete.

We conclude with another example.

Proposition 4.4. Let $1 \leq k \leq n, T: \mathbb{R}^{n} \rightarrow \mathbb{R}^{n}$ be a symmetric linear operator and $T^{*}: \Lambda^{k} \rightarrow \Lambda^{k}$ be the pullback of $T$. Let $f: \Lambda^{k} \rightarrow \mathbb{R}$ be defined as

$$
f(\xi)=\left\langle T^{*}(\xi) ; \xi\right\rangle, \text { for every } \xi \in \Lambda^{k}
$$

Then $f$ is ext. one convex if and only if $f$ is convex.

Proof. Since convexity implies ext. one convexity, we only have to prove the reverse implication.

Step 1. Since $T$ is symmetric, we can find eigenvalues $\left\{\lambda_{1}, \ldots, \lambda_{n}\right\}$ (not necessarily distinct) of $T$ with a corresponding set of orthonormal eigenvectors $\left\{\varepsilon^{1}, \ldots, \varepsilon^{n}\right\}$. Let 
$\left\{e^{1}, \ldots, e^{n}\right\}$ be the standard basis of $\mathbb{R}^{n}$ and let $\Lambda=\operatorname{diag}\left(\lambda_{1}, \ldots, \lambda_{n}\right)$ and $Q$ be the orthogonal matrix so that

$$
Q^{*}\left(\varepsilon^{i}\right)=e^{i}, \quad \text { for } i=1, \ldots, n .
$$

In terms of matrices what we have written just means that

$$
T=Q \Lambda Q^{t}
$$

Observe that, for every $i=1, \ldots, n$,

$$
T^{*}\left(\varepsilon^{i}\right)=\left(Q \Lambda Q^{t}\right)^{*}\left(\varepsilon^{i}\right)=\left(Q^{t}\right)^{*}\left(\Lambda^{*}\left(Q^{*}\left(\varepsilon^{i}\right)\right)\right)=\left(Q^{t}\right)^{*}\left(\Lambda^{*}\left(e^{i}\right)\right)=\lambda_{i} \varepsilon^{i} .
$$

This implies, for every $1 \leq k \leq n$ and $I \in \mathcal{T}_{k}^{n}$,

$$
T^{*}\left(\varepsilon^{I}\right)=T^{*}\left(\varepsilon^{i_{1}} \wedge \cdots \wedge \varepsilon^{i_{k}}\right)=T^{*}\left(\varepsilon^{i_{1}}\right) \wedge \cdots \wedge T^{*}\left(\varepsilon^{i_{k}}\right)=\left(\prod_{j=1}^{k} \lambda_{i_{j}}\right) \varepsilon^{I} .
$$

Step 2. Since $f$ is ext one convex and in view of Lemma4.1(iii), we have

$$
f\left(\varepsilon^{I}\right)=\left\langle\left(T^{*}\left(\varepsilon^{I}\right)\right) ; \varepsilon^{I}\right\rangle \geq 0
$$

and thus

$$
\prod_{j=1}^{k} \lambda_{i_{j}}=\prod_{i \in I} \lambda_{i} \geq 0
$$

Writing $\xi$ in the basis $\left\{\varepsilon^{1}, \ldots, \varepsilon^{n}\right\}$, we get

$$
f(\xi)=\left\langle T^{*}(\xi) ; \xi\right\rangle=\left\langle T^{*}\left(\sum_{I \in \mathcal{T}_{k}^{n}} \xi_{I} \varepsilon^{I}\right) ; \sum_{I \in \mathcal{T}_{k}^{n}} \xi_{I} \varepsilon^{I}\right\rangle=\sum_{I \in \mathcal{T}_{k}^{n}}\left(\prod_{i \in I} \lambda_{i}\right)\left(\xi_{I}\right)^{2},
$$

which according to (11) is non negative. This shows that $f$ is convex as wished.

\subsubsection{The main result}

We now turn to the main theorem.

Theorem 4.5. Let $1 \leq k \leq n, M: \Lambda^{k} \rightarrow \Lambda^{k}$ be a symmetric linear operator and $f: \Lambda^{k} \rightarrow \mathbb{R}$ be such that, for every $\xi \in \Lambda^{k}$,

$$
f(\xi)=\langle M \xi ; \xi\rangle .
$$

(i) The following equivalence holds in all cases

$$
\text { f ext. quasiconvex } \Leftrightarrow f \text { ext. one convex. }
$$


(ii) Let $k=2$. If $n=2$ or $n=3$, then

$f$ convex $\Leftrightarrow f$ ext. polyconvex $\Leftrightarrow f$ ext. quasiconvex $\Leftrightarrow f$ ext. one convex.

If $n=4$, then

$$
f \text { convex } \underset{\nLeftarrow}{\Rightarrow} \text { ext. polyconvex } \Leftrightarrow f \text { ext. quasiconvex } \Leftrightarrow f \text { ext. one convex, }
$$

while if $n \geq 6$, then

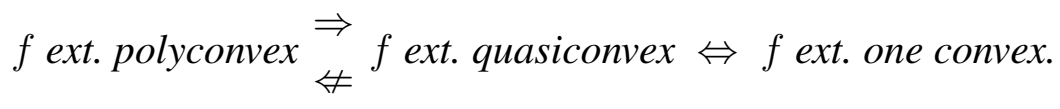

(iii) If $k$ is odd or if $2 k>n$, then

$$
f \text { convex } \Leftrightarrow f \text { ext. polyconvex. }
$$

(iv) If $k$ is even and $2 k \leq n$, then

$$
\text { f convex } \underset{\nLeftarrow}{\Rightarrow} \text { ext. polyconvex. }
$$

(v) If either $3 \leq k \leq n-3$ or $k=n-2 \geq 4$ is even, then

$$
\text { f ext. polyconvex } \underset{\nLeftarrow}{\Rightarrow} \text { ext. quasiconvex } \Leftrightarrow f \text { ext. one convex. }
$$

Remark 4.6. (i) We recall that when $k=1$ all notions of convexity are equivalent.

(ii) When $k=2$ and $n=5$, the equivalence between ext. polyconvexity and ext. quasiconvexity remains open.

Proof. (i) The result follows from Lemma 4.1 and Plancherel formula. The proof is similar to that of the case of the gradient (cf. Theorem 5.25 and Lemma 5.28 in [8]).

(ii) If $n=2$ or $n=3$, the result follows from Theorem 2.8(ii). If $n \geq 6$, see Theorem 4.7. So we now assume that $n=4$ (for the counter implication see (iv) below). We only have to prove that

$$
f \text { ext. one convex } \Rightarrow f \text { ext. polyconvex. }
$$

We know (by ext. one convexity) that, for every $a, b \in \Lambda^{1}\left(\mathbb{R}^{4}\right)$

$$
f(a \wedge b) \geq 0
$$

and we wish to show (cf. Lemma 4.1) that we can find $\alpha \in \Lambda^{4}\left(\mathbb{R}^{4}\right)$ so that

$$
f(\xi) \geq\langle\alpha ; \xi \wedge \xi\rangle
$$

Step 1. Let us change slightly the notations and write $\xi \in \Lambda^{2}\left(\mathbb{R}^{4}\right)$ as a vector of $\mathbb{R}^{6}$ in the following manner

$$
\xi=\left(\xi_{12}, \xi_{13}, \xi_{14}, \xi_{23}, \xi_{24}, \xi_{34}\right)
$$


and therefore $f$ can be seen as a quadratic form over $\mathbb{R}^{6}$ which is non-negative whenever the quadratic form (note also that $g$ is indefinite)

$$
g(\xi)=\left\langle e^{1} \wedge e^{2} \wedge e^{3} \wedge e^{4} ; \xi \wedge \xi\right\rangle=2\left(\xi_{12} \xi_{34}-\xi_{13} \xi_{24}+\xi_{14} \xi_{23}\right)
$$

vanishes. Indeed note that, by Proposition 2.37 in [5],

$$
g(\xi)=0 \Leftrightarrow \xi \wedge \xi=0 \Leftrightarrow \operatorname{rank}[\xi] \in\{0,2\} .
$$

By Proposition 2.43 in [5], this last condition is equivalent to the existence of $a, b \in$ $\Lambda^{1}\left(\mathbb{R}^{4}\right)$ so that

$$
\xi=a \wedge b,
$$

and by ext. one convexity we know that $f(a \wedge b) \geq 0$.

Step 2. We now invoke Theorem 2 in [12] to find $\lambda \in \mathbb{R}$ such that

$$
f(\xi)-\lambda g(\xi) \geq 0
$$

But this is exactly what we had to prove.

(iii) This is a general fact. (See Remark 2.2(ii) and Theorem 2.8)

(iv) The counterexample is just

$$
f(\xi)=\langle\alpha ; \xi \wedge \xi\rangle
$$

for any $\alpha \in \Lambda^{2 k}, \alpha \neq 0$.

(v) This is just Proposition 4.2 and the remark following it. Indeed we consider the two following cases.

- If $k$ is odd (and since $3 \leq k \leq n-3$, then $n \geq 6$ ), we know from (iii) that $f$ is ext. polyconvex if and only if $f$ is convex and we also know that there exists an exterior $k$-form which is not 1-divisible. Proposition 4.2 gives therefore the result.

- If $k$ is even and $4 \leq k \leq n-2$ (which implies again $n \geq 6$ ), then there exists an exterior $k$-form $\alpha$ which is not 1-divisible, but $\alpha \wedge \alpha=0$. The result thus follows again by Proposition 4.2 .

\subsubsection{A counterexample for $k=2$}

We now turn to a counterexample that has been mentioned in Theorem 4.5 ,

Theorem 4.7. Let $n \geq 6$. Then there exists a quadratic form $f: \Lambda^{2} \rightarrow \mathbb{R}$ which is ext. one convex but not ext. polyconvex. 
Proof. It is enough to establish the theorem for $n=6$. Our counterexample is inspired by Serre [16] and Terpstra [22] (see Theorem 5.25 (iii) in [8]). It is more convenient to write here $\xi \in \Lambda^{2}\left(\mathbb{R}^{6}\right)$ as

$$
\xi=\sum_{1 \leq i<j \leq 6} \xi_{j}^{i} e^{i} \wedge e^{j}
$$

So let

$$
g(\xi)=\left(\xi_{2}^{1}\right)^{2}+\left(\xi_{3}^{1}\right)^{2}+\left(\xi_{3}^{2}\right)^{2}+\left(\xi_{5}^{4}\right)^{2}+\left(\xi_{6}^{4}\right)^{2}+\left(\xi_{6}^{5}\right)^{2}+h(\xi)
$$

where

$$
h(\xi)=\left(\xi_{4}^{1}-\xi_{5}^{3}-\xi_{6}^{2}\right)^{2}+\left(\xi_{5}^{1}-\xi_{4}^{3}+\xi_{6}^{1}\right)^{2}+\left(\xi_{4}^{2}-\xi_{4}^{3}-\xi_{6}^{1}\right)^{2}+\left(\xi_{5}^{2}\right)^{2}+\left(\xi_{6}^{3}\right)^{2} .
$$

Note that $g \geq 0$. We claim that there exists $\gamma>0$ so that

$$
f(\xi)=g(\xi)-\gamma|\xi|^{2}
$$

is ext. one convex (cf. Step 1) but not ext. polyconvex (cf. Step 2).

Step 1. Define

$$
\gamma:=\inf \left\{g(a \wedge b): a, b \in \Lambda^{1}\left(\mathbb{R}^{6}\right),|a \wedge b|=1\right\}
$$

Note that $\gamma \geq 0$, and it follows from Lemma 4.1 that $f$ is ext. one convex.

Let us claim that, in fact $\gamma>0$, which will imply in Step 2 that $f$ is not ext. polyconvex. To show that $\gamma>0$, we proceed by contradiction and assume that $\gamma=0$. This implies that we can find $a, b \in \Lambda^{1}\left(\mathbb{R}^{6}\right)$ with $|a \wedge b|=1$ such that

$$
\begin{gathered}
\left\{\begin{array} { l } 
{ a ^ { 1 } b _ { 2 } - a ^ { 2 } b _ { 1 } = 0 } \\
{ a ^ { 1 } b _ { 3 } - a ^ { 3 } b _ { 1 } = 0 } \\
{ a ^ { 2 } b _ { 3 } - a ^ { 3 } b _ { 2 } = 0 }
\end{array} \quad \left\{\begin{array} { l } 
{ a ^ { 4 } b _ { 5 } - a ^ { 5 } b _ { 4 } = 0 } \\
{ a ^ { 4 } b _ { 6 } - a ^ { 6 } b _ { 4 } = 0 } \\
{ a ^ { 5 } b _ { 6 } - a ^ { 6 } b _ { 5 } = 0 }
\end{array} \quad \left\{\begin{array}{l}
a^{2} b_{5}-a^{5} b_{2}=0 \\
a^{3} b_{6}-a^{6} b_{3}=0
\end{array}\right.\right.\right. \\
\left\{\begin{array}{l}
\left(a^{1} b_{4}-a^{4} b_{1}\right)-\left(a^{3} b_{5}-a^{5} b_{3}\right)-\left(a^{2} b_{6}-a^{6} b_{2}\right)=0 \\
\left(a^{1} b_{5}-a^{5} b_{1}\right)-\left(a^{3} b_{4}-a^{4} b_{3}\right)+\left(a^{1} b_{6}-a^{6} b_{1}\right)=0 \\
\left(a^{2} b_{4}-a^{4} b_{2}\right)-\left(a^{3} b_{4}-a^{4} b_{3}\right)-\left(a^{1} b_{6}-a^{6} b_{1}\right)=0 .
\end{array}\right.
\end{gathered}
$$

Let us introduce some notation, we write

$$
\underline{a}=\left(\begin{array}{c}
a^{1} \\
a^{2} \\
a^{3}
\end{array}\right), \quad \underline{b}=\left(\begin{array}{c}
b_{1} \\
b_{2} \\
b_{3}
\end{array}\right), \quad \bar{a}=\left(\begin{array}{c}
a^{4} \\
a^{5} \\
a^{6}
\end{array}\right), \quad \bar{b}=\left(\begin{array}{c}
b_{4} \\
b_{5} \\
b_{6}
\end{array}\right) .
$$

Note that the first and second sets of equations lead to

$$
\underline{a} \| \underline{b} \text { and } \bar{a} \| \bar{b}
$$

We consider two cases starting with the generic case. 
Case 1: there exist $\lambda, \mu \in \mathbb{R}$ such that

$$
\underline{a}=\lambda \underline{b} \quad \text { and } \quad \bar{a}=\mu \bar{b}
$$

(The same reasoning applies to all other cases, for example $\underline{b}=\lambda \underline{a}$ and $\bar{b}=\mu \bar{a}$ ). Note that $\lambda \neq \mu$, otherwise we would have $a=\lambda b$ and thus $a \wedge b=0$ contradicting the fact that $|a \wedge b|=1$. Inserting this in the third and fourth sets of equations we get

$$
\left\{\begin{array} { l } 
{ ( \lambda - \mu ) b _ { 2 } b _ { 5 } = 0 } \\
{ ( \lambda - \mu ) b _ { 3 } b _ { 6 } = 0 }
\end{array} \quad \left\{\begin{array}{l}
(\lambda-\mu)\left[b_{1} b_{4}-b_{3} b_{5}-b_{2} b_{6}\right]=0 \\
(\lambda-\mu)\left[b_{1} b_{5}-b_{3} b_{4}+b_{1} b_{6}\right]=0 \\
(\lambda-\mu)\left[b_{2} b_{4}-b_{3} b_{4}-b_{1} b_{6}\right]=0
\end{array}\right.\right.
$$

and thus, since $\lambda \neq \mu$,

$$
b_{2} b_{5}=b_{3} b_{6}=b_{1} b_{4}-b_{3} b_{5}-b_{2} b_{6}=b_{1} b_{5}-b_{3} b_{4}+b_{1} b_{6}=b_{2} b_{4}-b_{3} b_{4}-b_{1} b_{6}=0
$$

We have to consider separately the cases $b_{2}=b_{3}=0, b_{5}=b_{6}=0, b_{2}=b_{6}=0$ and $b_{3}=b_{5}=0$. We do only the first case. Other cases can be handled similarly. Let us assume that $b_{2}=b_{3}=0$. We thus have

$$
b_{1} b_{4}=b_{1} b_{5}+b_{1} b_{6}=b_{1} b_{6}=0 \text {. }
$$

So either $b_{1}=0$ and thus $\underline{b}=0$ and hence $\underline{a}=0$ and again this implies that $a=\mu b$ which contradicts the fact that $|a \wedge b|=1$. Or $b_{4}=b_{5}=b_{6}=0$ and thus $\bar{b}=\bar{a}=0$ which as before contradicts the fact that $|a \wedge b|=1$.

Case $2: \underline{b}=0$ and $\bar{a}=0$ (or $\underline{a}=0$ and $\bar{b}=0$ which is handled similarly). This means that $a^{4}=a^{5}=a^{6}=0$ and $b_{1}=b_{2}=b_{3}=0$. We therefore have

$$
a^{2} b_{5}=a^{3} b_{6}=a^{1} b_{4}-a^{3} b_{5}-a^{2} b_{6}=a^{1} b_{5}-a^{3} b_{4}+a^{1} b_{6}=a^{2} b_{4}-a^{3} b_{4}-a^{1} b_{6}=0
$$

Four cases can happen $a^{2}=a^{3}=0, a^{2}=b_{6}=0, a^{3}=b_{5}=0$ and $b_{5}=b_{6}=0$. Again, we handle only the first case. Other cases can be treated similarly. Assuming that $a^{2}=a^{3}=0$, we have

$$
a^{1} b_{4}=a^{1} b_{5}+a^{1} b_{6}=a^{1} b_{6}=0 .
$$

So either $a^{1}=0$ and thus $a=0$ which is impossible. Or $b_{4}=b_{5}=b_{6}=0$ and thus $b=0$ which again cannot happen. Hence, we have proved that $\gamma$ defined in Equation (12) is positive.

Step 2. We now show that $f$ is not ext. polyconvex. In view of Lemma 4.1 (i), it is sufficient to show that for every $\alpha \in \Lambda^{4}\left(\mathbb{R}^{6}\right)$, there exists $\xi \in \Lambda^{2}\left(\mathbb{R}^{6}\right)$ such that

$$
f(\xi)+\frac{1}{2}\langle\alpha ; \xi \wedge \xi\rangle<0
$$


We prove that the above inequality holds for forms $\xi$ of the following form

$$
\begin{aligned}
\xi & =(b+d) e^{1} \wedge e^{4}+(c-a) e^{1} \wedge e^{5}+a e^{1} \wedge e^{6} \\
& +(c+a) e^{2} \wedge e^{4}+b e^{2} \wedge e^{6}+c e^{3} \wedge e^{4}+d e^{3} \wedge e^{5} .
\end{aligned}
$$

Note that

$$
\begin{aligned}
\frac{1}{2} \xi \wedge \xi & =\left(c^{2}-a^{2}\right) e^{1} \wedge e^{2} \wedge e^{4} \wedge e^{5}+\left(a c+a^{2}-b^{2}-b d\right) e^{1} \wedge e^{2} \wedge e^{4} \wedge e^{6} \\
& +(a b-b c) e^{1} \wedge e^{2} \wedge e^{5} \wedge e^{6}+\left(c^{2}-a c-b d-d^{2}\right) e^{1} \wedge e^{3} \wedge e^{4} \wedge e^{5} \\
& +a c e^{1} \wedge e^{3} \wedge e^{4} \wedge e^{6}+a d e^{1} \wedge e^{3} \wedge e^{5} \wedge e^{6} \\
& +(-c d-a d) e^{2} \wedge e^{3} \wedge e^{4} \wedge e^{5}+b c e^{2} \wedge e^{3} \wedge e^{4} \wedge e^{6} \\
& +b d e^{2} \wedge e^{3} \wedge e^{5} \wedge e^{6}
\end{aligned}
$$

For such forms we have $g(\xi)=0$ and therefore

$$
f(\xi)=-\gamma|\xi|^{2}=-\gamma\left[(b+d)^{2}+(c-a)^{2}+a^{2}+(c+a)^{2}+b^{2}+c^{2}+d^{2}\right] .
$$

Moreover,

$$
\begin{aligned}
\frac{1}{2}\langle\alpha ; \xi \wedge \xi\rangle & =\alpha_{1245}\left(c^{2}-a^{2}\right)+\alpha_{1246}\left(a c+a^{2}-b^{2}-b d\right) \\
& +\alpha_{1256}(a b-b c)+\alpha_{1345}\left(c^{2}-a c-b d-d^{2}\right)+\alpha_{1346} a c \\
& +\alpha_{1356} a d+\alpha_{2345}(-c d-a d)+\alpha_{2346} b c+\alpha_{2356} b d
\end{aligned}
$$

We consider three cases.

Case 1. If $\alpha_{1246}>0$, then take $a=c=d=0$ and $b \neq 0$, to get

$$
f(\xi)+\frac{1}{2}\langle\alpha ; \xi \wedge \xi\rangle=-\gamma\left(2 b^{2}\right)-\alpha_{1246} b^{2}<0 .
$$

Case 2. If $\alpha_{1345}>0$, then take $a=b=c=0$ and $d \neq 0$, to get

$$
f(\xi)+\frac{1}{2}\langle\alpha ; \xi \wedge \xi\rangle=-\gamma\left(2 d^{2}\right)-\alpha_{1345} d^{2}<0 .
$$

We therefore can assume that $\alpha_{1246} \leq 0$ and $\alpha_{1345} \leq 0$.

Case 3. If $\alpha_{1245}+\alpha_{1345}<0\left(\alpha_{1246} \leq 0, \alpha_{1345} \leq 0\right)$, then take $a=b=d=0$ and $c \neq 0$ to get

$$
f(\xi)+\frac{1}{2}\langle\alpha ; \xi \wedge \xi\rangle=-\gamma\left(3 c^{2}\right)+\left(\alpha_{1245}+\alpha_{1345}\right) c^{2}<0 .
$$

We therefore assume $\alpha_{1246} \leq 0, \alpha_{1345} \leq 0$ and $\alpha_{1245}+\alpha_{1345} \geq 0$. From these three inequalities we deduce that $\alpha_{1246}-\alpha_{1245} \leq 0$, and then taking $b=c=d=0$ and $a \neq 0$, we get

$$
f(\xi)+\frac{1}{2}\langle\alpha ; \xi \wedge \xi\rangle=-\gamma\left(3 a^{2}\right)+\left(\alpha_{1246}-\alpha_{1245}\right) a^{2}<0 .
$$

This concludes the proof of the theorem. 


\subsection{Ext. one convexity does not imply ext. quasiconvexity}

We now give an important counterexample for any $k \geq 2$. It is an adaptation of the fundamental result of Sverak [20] (see also Theorem 5.50 in [8]).

Theorem 4.8. Let $2 \leq k \leq n-3$. Then there exists $f: \Lambda^{k} \rightarrow \mathbb{R}$ ext. one convex but not ext. quasiconvex.

Remark 4.9. We know that when $k=1, n-1, n$ or $k=n-2$ is odd, then

$f$ convex $\Leftrightarrow f$ ext. polyconvex $\Leftrightarrow f$ ext. quasiconvex $\Leftrightarrow f$ ext. one convex.

Therefore only the case $k=n-2 \geq 2$ even (including $k=2$ and $n=4$ ) remains open.

The main algebraic tool in order to adapt Sverak example is given in the following lemma.

Lemma 4.10. Let $k \geq 2$ and $n=k+3$. There exist

$$
\alpha, \beta, \gamma \in \operatorname{span}\left\{e^{i_{1}} \wedge \cdots \wedge e^{i_{k-1}}, \quad 3 \leq i_{1}<\cdots<i_{k-1} \leq k+3\right\} \subset \Lambda^{k-1}\left(\mathbb{R}^{k+3}\right)
$$

such that if

$$
L=\operatorname{span}\left\{e^{1} \wedge \alpha, e^{2} \wedge \beta,\left(e^{1}+e^{2}\right) \wedge \gamma\right\},
$$

then any 1-divisible $\xi=(x, y, z)=x e^{1} \wedge \alpha+y e^{2} \wedge \beta+z\left(e^{1}+e^{2}\right) \wedge \gamma \in L$ necessarily verifies

$$
x y=x z=y z=0 .
$$

Proof. Step 1. We choose, recall that $n=k+3$,

$$
\begin{gathered}
\alpha= \begin{cases}\sum_{i=2}^{l+1}\left(\widehat{e^{2 i}} \wedge \widehat{e^{2 i+1}}\right) & \text { if } k=2 l, \\
\sum_{i=2}^{l+2}\left(\widehat{e^{2 i-1}} \wedge \widehat{e^{2 i}}\right) & \text { if } k=2 l+1 .\end{cases} \\
\beta=\left\{\begin{array}{cc}
\widehat{e^{3}} \wedge e^{2 l+3} & \text { if } k=2 l, \\
\left(\widehat{e^{3}} \wedge \widehat{e^{5}}+\left(\widehat{e^{4}} \wedge \widehat{e^{6}}\right)\right. & \text { if } k=3, \\
\sum_{i=2}^{l}\left(\widehat{e^{2 i-1}} \wedge \widehat{e^{2 i}}\right) & \text { if } k=2 l+1 \text { and } k \geq 5 .
\end{array}\right. \\
\gamma=\left\{\begin{array}{cc}
\sum_{i=2}^{l+1}\left(\widehat{e^{2 i-1}} \wedge \widehat{e^{2 i}}\right) & \text { if } k=2 l, \\
\left(\widehat{e^{2 l+1}} \wedge \widehat{e^{2 l+4}}\right)+\left(\widehat{e^{2 l+2}} \wedge \widehat{e^{2 l+3}}\right) & \text { if } k=2 l+1,
\end{array}\right.
\end{gathered}
$$

where we write, by abuse of notations, for $3 \leq i<j \leq k+3$,

$$
\widehat{e^{i}} \wedge \widehat{e^{j}}=e^{3} \wedge \cdots \wedge \widehat{e^{i}} \wedge \cdots \wedge \widehat{e^{j}} \wedge \cdots \wedge e^{k+3}
$$


Observe that $\{\alpha, \beta, \gamma\}$ are linearly independent.

Step 2. We now prove the statement, namely that if $\xi=(x, y, z) \in L$ is 1-divisible (i.e. $\xi=b \wedge a$ for $a \in \Lambda^{1}$ and $b \in \Lambda^{k-1}$ ), then necessarily

$$
x y=x z=y z=0 .
$$

Assume that $\xi \neq 0$ (otherwise the result is trivial) and thus $a \neq 0$. Note that if $\xi=b \wedge a$, then $a \wedge \xi=0$. We write

$$
a=\sum_{i=1}^{k+3} a_{i} e^{i} \neq 0 .
$$

Step 2.1. Since $a \wedge \xi=0$ we deduce that the term involving $e^{1} \wedge e^{2}$ must be 0 and thus

$$
-a_{2} x \alpha+a_{1} y \beta+\left(a_{1}-a_{2}\right) z \gamma=0
$$

Since $\{\alpha, \beta, \gamma\}$ are linearly independent, we deduce that

$$
a_{2} x=a_{1} y=\left(a_{1}-a_{2}\right) z=0 .
$$

From there we infer that $x y=x z=y z=0$, as soon as either $a_{1} \neq 0$ or $a_{2} \neq 0$. So in order to establish the lemma it is enough to consider $a$ of the form

$$
a=\sum_{i=3}^{k+3} a_{i} e^{i} \neq 0
$$

We therefore have

$$
\sum_{i=3}^{k+3} a_{i} e^{i} \wedge\left[e^{1} \wedge(x \alpha+z \gamma)+e^{2} \wedge(y \beta+z \gamma)\right]=0
$$

which implies that

$$
\left\{\begin{array}{l}
a \wedge(x \alpha+z \gamma)=\sum_{i=3}^{k+3} a_{i} e^{i} \wedge(x \alpha+z \gamma)=0 \\
a \wedge(y \beta+z \gamma)=\sum_{i=3}^{k+3} a_{i} e^{i} \wedge(y \beta+z \gamma)=0 .
\end{array}\right.
$$

We continue the discussion considering separately the cases $k$ even, $k=3$ and $k \geq 5$ odd. They are all treated in the same way and we prove it only in the even case.

Step 2.2: $k=2 l \geq 2$. We have to prove that if

$$
a=\sum_{i=3}^{2 l+3} a_{i} e^{i} \neq 0
$$

satisfies (13), then necessarily

$$
x y=x z=y z=0 .
$$


We find (up to a + or - sign but here it is immaterial)

$$
\begin{aligned}
& a \wedge \alpha=\sum_{i=2}^{l+1}\left(a_{2 i+1} \widehat{e^{2 i}}\right)+\sum_{i=2}^{l}\left(a_{2 i} \widehat{e^{2 i+1}}\right)+a_{2 l+2} \widehat{e^{2 l+3}} \\
& a \wedge \beta=a_{2 l+3} \widehat{e^{3}}+a_{3} \widehat{e^{2 l+3}} \\
& a \wedge \gamma=a_{4} \widehat{e^{3}}+\sum_{i=2}^{l+1}\left(a_{2 i-1} \widehat{e^{2 i}}\right)+\sum_{i=2}^{l}\left(a_{2 i+2} \widehat{e^{2 i+1}}\right) .
\end{aligned}
$$

Therefore,

$$
\begin{aligned}
a \wedge(x \alpha+z \gamma)= & z a_{4} \widehat{e^{3}}+\sum_{i=2}^{l+1}\left(x a_{2 i+1}+z a_{2 i-1}\right) \widehat{e^{2 i}} \\
& +\sum_{i=2}^{l}\left(x a_{2 i}+z a_{2 i+2}\right) \widehat{e^{2 i+1}}+x a_{2 l+2} \widehat{e^{2 l+3}} \\
a \wedge(y \beta+z \gamma)= & \left(y a_{2 l+3}+z a_{4}\right) \widehat{e^{3}}+z\left\{\sum_{i=2}^{l+1}\left(a_{2 i-1} \widehat{e^{2 i}}\right)+\sum_{i=2}^{l}\left(a_{2 i+2} \widehat{e^{2 i+1}}\right)\right\} \\
& +y a_{3} \widehat{e^{2 l+3}} .
\end{aligned}
$$

Case 1:x=z=0. This is our claim.

Case $2: z=0$ and $x \neq 0$. We can also assume that $y \neq 0$ otherwise we have the claim $y=z=0$. From the first equation we obtain

$$
a_{2 i}=a_{2 i+1}=0, \quad i=2, \cdots, l+1 .
$$

So only $a_{3}$ might be non-zero. However since $y \neq 0$ we deduce from the second equation that $a_{3}=0$ and thus $a=0$ which is impossible.

Case $3: x=0$ and $z \neq 0$. We can also assume that $y \neq 0$ otherwise we have the claim $x=y=0$. From the first equation we obtain

$$
a_{2 i}=a_{2 i-1}=0, \quad i=2, \cdots, l+1
$$

So only $a_{2 l+3}$ might be non-zero. However since $y \neq 0$ we deduce, appealing to the second equation, that $a_{2 l+3}=0$ and thus $a=0$ which is again impossible.

Case $4: x z \neq 0$. From the first equation we deduce that

$$
a_{2 i}=0, \quad i=2, \cdots, l+1
$$

Inserting this in the second equation we get

$$
a \wedge(y \beta+z \gamma)=y a_{2 l+3} \widehat{e^{3}}+z \sum_{i=2}^{l+1}\left(a_{2 i-1} \widehat{e^{2 i}}\right)+y a_{3} \widehat{e^{2 l+3}}
$$


Since $z \neq 0$, we infer that

$$
a_{2 i-1}=0, \quad i=2, \cdots, l+1
$$

So only $a_{2 l+3}$ might be non-zero. However returning to the first equation we have

$$
x a_{2 l+3}=0 .
$$

But since $x \neq 0$, we deduce that $a_{2 l+3}=0$ and thus $a=0$ which is again impossible. This settles the case $k$ even. The odd case is handled in a very similar manner and we leave out the details

We may now conclude with the proof of Theorem 4.8, which is, once the above lemma is established, almost identical to the proof of Sverak.

Proof. It is enough to prove the theorem for $n=k+3$.

Step 1. We start with some notations. Let $L$ be as in Lemma 4.10, An element $\xi$ of $L$ is, when convenient, denoted by $\xi=(x, y, z) \in L$. Recall that if $\xi=(x, y, z) \in L$ is 1-divisible, meaning that $\xi=b \wedge a$ for a certain $a \in \Lambda^{1}$ and $b \in \Lambda^{k-1}$, then necessarily

$$
x y=x z=y z=0 .
$$

We next let $P: \Lambda^{k}\left(\mathbb{R}^{k+3}\right) \rightarrow L$ be the projection map; in particular $P(\xi)=\xi$ if $\xi \in L$.

Step 2. Let $g: L \subset \Lambda^{k}\left(\mathbb{R}^{k+3}\right) \rightarrow \mathbb{R}$ be defined by

$$
g(\xi)=-x y z
$$

Observe that, $g$ is ext. one affine when restricted to $L$. Indeed, if $\xi=(x, y, z) \in L$ and $\eta=(a, b, c) \in L$ is 1 -divisible (which implies that $a b=a c=b c=0$ ), then

$$
g(\xi+t \eta)=-(x+t a)(y+t b)(z+t c)=-x y z-t[x y c+x z b+y z a] .
$$

We therefore have that, for every $\xi, \eta \in L$ with $\eta$ being 1-divisible,

$$
L_{g}(\xi, \eta)=\left.\frac{d^{2}}{d t^{2}} g(\xi+t \eta)\right|_{t=0}=0 .
$$

Step 3. Let $\omega \in C_{\text {per }}^{\infty}\left((0,2 \pi)^{k+3} ; \Lambda^{k-1}\right)$ be defined by

$$
\omega=\left(\sin x_{1}\right) \alpha+\left(\sin x_{2}\right) \beta+\left(\sin \left(x_{1}+x_{2}\right)\right) \gamma,
$$

so that

$$
d \omega=\left(\cos x_{1}\right) d x^{1} \wedge \alpha+\left(\cos x_{2}\right) d x^{2} \wedge \beta+\left(\cos \left(x_{1}+x_{2}\right)\right)\left(d x^{1}+d x^{2}\right) \wedge \gamma
$$


and hence $d \omega \in L$. Note that

$$
\int_{0}^{2 \pi} \int_{0}^{2 \pi} g(d \omega) d x_{1} d x_{2}=-\int_{0}^{2 \pi} \int_{0}^{2 \pi}\left(\cos x_{1}\right)^{2}\left(\cos x_{2}\right)^{2} d x_{1} d x_{2}<0 .
$$

Step 4. Assume, cf. Step 5, that we have shown that for every $\epsilon>0$, we can find $\gamma=\gamma(\epsilon)>0$ such that

$$
f_{\epsilon}(\xi)=g(P(\xi))+\epsilon|\xi|^{2}+\epsilon|\xi|^{4}+\gamma|\xi-P(\xi)|^{2}
$$

is ext. one convex. Then noting that

$$
f_{\epsilon}(d \omega)=g(d \omega)+\epsilon|d \omega|^{2}+\epsilon|d \omega|^{4}
$$

we deduce from Step 3 that, for $\epsilon>0$ small enough

$$
\int_{(0,2 \pi)^{k+3}} f_{\epsilon}(d \omega) d x<0 .
$$

This shows that $f_{\epsilon}$ is not ext. quasiconvex. The proposition is therefore proved.

Step 5. It remains to prove that for every $\epsilon>0$ we can find $\gamma=\gamma(\epsilon)>0$ such that

$$
f_{\epsilon}(\xi)=g(P(\xi))+\epsilon|\xi|^{2}+\epsilon|\xi|^{4}+\gamma|\xi-P(\xi)|^{2}
$$

is ext. one convex. This is equivalent to showing that, for every $\xi, \eta \in \Lambda^{k}$ with $\eta$ being 1-divisible,

$$
\begin{aligned}
L_{f_{\epsilon}}(\xi, \eta) & =\left.\frac{d^{2}}{d t^{2}} f_{\epsilon}(\xi+t \eta)\right|_{t=0} \\
& =L_{g}(P(\xi), P(\eta))+2 \epsilon|\eta|^{2}+4 \epsilon|\xi|^{2}|\eta|^{2}+8 \epsilon(\langle\xi ; \eta\rangle)^{2}+2 \gamma|\eta-P(\eta)|^{2} \\
& \geq 0 .
\end{aligned}
$$

The proof follows in the standard way, see [8] and [17] for more detail.

\subsection{Some further examples}

We here give another counterexample for $k=2$.

Proposition 4.11. Let $n \geq 4$. Then there exists an ext. quasiconvex function $f: \Lambda^{2}\left(\mathbb{R}^{n}\right) \rightarrow$ $\mathbb{R}$ which is not ext. polyconvex.

Remark 4.12. This example is mostly interesting when $n=4$ or 5 . Since when $n \geq 6$, we already have such a counterexample (cf. Theorem 4.7). 
Proof. As in previous theorems, it is easy to see that it is enough to establish the theorem for $n=4$. Let $1<p<2, \alpha=e^{1} \wedge e^{2}+e^{3} \wedge e^{4}$ and $g: \Lambda^{2}\left(\mathbb{R}^{4}\right) \rightarrow \mathbb{R}$ be given by

$$
g(\xi)=\left(|\xi|^{2}-2|\langle\alpha ; \xi\rangle|+|\alpha|^{2}\right)^{p / 2}=\min \left\{|\xi-\alpha|^{p},|\xi+\alpha|^{p}\right\}
$$

The claim is that $f=Q_{\text {ext }} g$ has all the desired properties (the proof is inspired by the one of Sverak [19], see also Theorem 5.54 in [8]). Indeed $f$ is by construction ext. quasiconvex and if we can show (cf. Step 2) that $f$ is not convex (note that $f$ is subquadratic and using Proposition 2.14, any subquadratic ext. polyconvex function is convex) we will have established the proposition.

Step 1. First observe that a direct computation gives

$$
|\xi|^{2}-2|\langle\alpha ; \xi\rangle|+|\alpha|^{2}=\min \left\{|\xi-\alpha|^{2},|\xi+\alpha|^{2}\right\} \geq \frac{1}{2}\left[|\xi|^{2}-\frac{1}{2}\langle\alpha \wedge \alpha ; \xi \wedge \xi\rangle\right] \geq 0
$$

We therefore get that there exists a constant $c_{1}>0$ such that

$$
\begin{aligned}
g(\xi) & \geq\left(\frac{1}{2}\right)^{\frac{p}{2}}\left[|\xi|^{2}-\frac{1}{2}\langle\alpha \wedge \alpha ; \xi \wedge \xi\rangle\right]^{p / 2} \\
& \geq c_{1}\left[\left|\xi_{12}-\xi_{34}\right|^{p}+\left|\xi_{13}+\xi_{24}\right|^{p}+\left|\xi_{14}-\xi_{23}\right|^{p}\right]
\end{aligned}
$$

Call $h$ the right hand side, namely

$$
h(\xi)=c_{1}\left[\left|\xi_{12}-\xi_{34}\right|^{p}+\left|\xi_{13}+\xi_{24}\right|^{p}+\left|\xi_{14}-\xi_{23}\right|^{p}\right] .
$$

Step 2. To prove that $f$ is not convex, we proceed by contradiction. Let us suppose, on the contrary, that $f$ is convex. This implies that $f(0)=0$, because

$$
0 \leq f(0)=f\left(\frac{1}{2} \alpha+\frac{1}{2}(-\alpha)\right) \leq \frac{1}{2} f(\alpha)+\frac{1}{2} f(-\alpha)=0 .
$$

Use Remark 2.13 to find a sequence of $\omega_{s} \in W_{\delta, T}^{1, \infty}\left(\Omega ; \Lambda^{1}\right)$ (we can choose an $\Omega$ with smooth boundary and by density we can also assume that $\left.\omega_{s} \in C_{\delta, T}^{\infty}\left(\bar{\Omega} ; \Lambda^{1}\right)\right)$ such that

$$
0 \leq \frac{1}{\operatorname{meas} \Omega} \int_{\Omega} g\left(d \omega_{s}\right) \leq Q_{e x t} g(0)+\frac{1}{s}=f(0)+\frac{1}{s}=\frac{1}{s},
$$

which implies that

$$
\lim _{s \rightarrow \infty} \frac{1}{\operatorname{meas} \Omega} \int_{\Omega} g\left(d \omega_{s}\right)=0
$$

On the other hand, from Step 1, we deduce that

$$
0 \leq \int_{\Omega} h\left(d \omega_{s}\right) \leq \frac{\text { meas } \Omega}{s} \rightarrow 0
$$


We now invoke Step 3 to find a constant $c_{2}>0$ such that

$$
c_{2}\left\|\nabla \omega_{s}\right\|_{L^{p}}^{p} \leq \int_{\Omega} h\left(d \omega_{s}\right) .
$$

Thus $\left\|d \omega_{s}\right\|_{L^{p}} \rightarrow 0$ and hence, up to the extraction of a subsequence,

$$
\frac{1}{\operatorname{meas} \Omega} \int_{\Omega} g\left(d \omega_{s}\right) \rightarrow g(0)=|\alpha|^{p} \neq 0,
$$

which contradicts Equation (14). Therefore, $f$ is not convex.

Step 3. It remains to prove that there exists a constant $\lambda>0$ such that

$$
\lambda\|\nabla \omega\|_{L^{p}}^{p} \leq \int_{\Omega} h(d \omega)=\left\|[h(d \omega)]^{1 / p}\right\|_{L^{p}}^{p}, \quad \text { for every } \omega \in C_{\delta, T}^{\infty}\left(\bar{\Omega} ; \Lambda^{1}\right) .
$$

To establish the estimate we proceed as follows. Let $\omega \in C_{\delta, T}^{\infty}\left(\bar{\Omega} ; \Lambda^{1}\right), \alpha, \beta, \gamma \in C^{\infty}(\bar{\Omega})$ be such that

$$
\begin{aligned}
& \alpha=(d \omega)_{12}-(d \omega)_{34}=-\omega_{x_{2}}^{1}+\omega_{x_{1}}^{2}+\omega_{x_{4}}^{3}-\omega_{x_{3}}^{4}, \\
& \beta=(d \omega)_{13}+(d \omega)_{24}=-\omega_{x_{3}}^{1}+\omega_{x_{1}}^{3}-\omega_{x_{4}}^{2}+\omega_{x_{2}}^{4}, \\
& \gamma=(d \omega)_{14}-(d \omega)_{23}=-\omega_{x_{4}}^{1}+\omega_{x_{1}}^{4}+\omega_{x_{3}}^{2}-\omega_{x_{2}}^{3} \\
& 0=\delta \omega
\end{aligned}
$$

Note that

$$
h(d \omega)=c_{1}\left[|\alpha|^{p}+|\beta|^{p}+|\gamma|^{p}\right] .
$$

Differentiating appropriately the four equations we find

$$
\left\{\begin{aligned}
\Delta \omega^{1} & =-\alpha_{x_{2}}-\beta_{x_{3}}-\gamma_{x_{4}}, \quad \Delta \omega^{2}=\alpha_{x_{1}}-\beta_{x_{4}}+\gamma_{x_{3}} \\
\Delta \omega^{3}=\alpha_{x_{4}}+\beta_{x_{1}}-\gamma_{x_{2}}, & \Delta \omega^{4}=-\alpha_{x_{3}}+\beta_{x_{2}}+\gamma_{x_{1}} .
\end{aligned}\right.
$$

Letting

$$
\begin{aligned}
\psi:= & -\left(\alpha_{x_{2}}+\beta_{x_{3}}+\gamma_{x_{4}}\right) d x^{1}+\left(\alpha_{x_{1}}-\beta_{x_{4}}+\gamma_{x_{3}}\right) d x^{2}+\left(\alpha_{x_{4}}+\beta_{x_{1}}-\gamma_{x_{2}}\right) d x^{3} \\
& +\left(-\alpha_{x_{3}}+\beta_{x_{2}}+\gamma_{x_{1}}\right) d x^{4},
\end{aligned}
$$

we get

$$
\left\{\begin{array}{cl}
\Delta \omega=\psi & \text { in } \Omega \\
\nu \wedge \delta \omega=0, \nu \wedge \omega=0 & \text { on } \partial \Omega .
\end{array}\right.
$$

Using classical elliptic regularity theory, see, for example, Theorem 6.3 .7 of [13], we deduce that

$$
\|\omega\|_{W^{1, p}} \leq \lambda_{2}\|\psi\|_{W^{-1, p}} .
$$

In other words,

$$
\|\nabla \omega\|_{L^{p}} \leq \lambda_{2}\|\psi\|_{W^{-1, p}} \leq \lambda_{3}\|(\alpha, \beta, \gamma)\|_{L^{p}} \leq \lambda_{4}\left\|[h(d \omega)]^{1 / p}\right\|_{L^{p}} .
$$

This is exactly what had to be proved. 


\section{Application to a minimization problem}

Theorem 5.1. Let $1 \leq k \leq n, p>1, \Omega \subset \mathbb{R}^{n}$ be a bounded smooth open set, $\omega_{0} \in$ $W^{1, p}\left(\Omega ; \Lambda^{k-1}\right)$ and $f: \Lambda^{k}\left(\mathbb{R}^{n}\right) \rightarrow \mathbb{R}$ be ext. quasiconvex verifying,

$$
c_{1}\left(|\xi|^{p}-1\right) \leq f(\xi) \leq c_{2}\left(|\xi|^{p}+1\right), \text { for every } \xi \in \Lambda^{k}
$$

for some $c_{1}, c_{2}>0$. Let

$$
\left(\mathcal{P}_{0}\right) \quad \inf \left\{\int_{\Omega} f(d \omega): \omega \in \omega_{0}+W_{0}^{1, p}\left(\Omega ; \Lambda^{k-1}\right)\right\}=m .
$$

Then the problem $\left(\mathcal{P}_{0}\right)$ has a minimizer.

Remark 5.2. (i) If

$$
\left(\mathcal{P}_{\delta, T}\right) \quad \inf \left\{\int_{\Omega} f(d \omega): \omega \in \omega_{0}+W_{\delta, T}^{1, p}\left(\Omega ; \Lambda^{k-1}\right)\right\}=m_{\delta, T}
$$

where $\omega_{0}+W_{\delta, T}^{1, p}\left(\Omega ; \Lambda^{k-1}\right)$ stands for the set of all $\omega \in W^{1, p}\left(\Omega ; \Lambda^{k-1}\right)$ such that

$$
\delta \omega=0 \text { in } \Omega \quad \text { and } \quad \nu \wedge \omega=\nu \wedge \omega_{0} \text { on } \partial \Omega,
$$

the proof of the theorem will show that $\left(\mathcal{P}_{\delta, T}\right)$ also has a minimizer and that $m_{\delta, T}=m$.

(ii) When the function $f$ is not ext. quasiconvex, in general the problem will not have a solution. However, in many cases it does have one, but the argument is of a different nature and uses results on differential inclusions, see [1], [2] and [9].

Proof. Step 1. Using a variant of the classical result, see [3] and [17], we note that if

$$
\alpha_{s} \rightarrow \alpha \quad \text { in } W^{1, p}\left(\Omega ; \Lambda^{k-1}\right)
$$

then

$$
\liminf _{s \rightarrow \infty} \int_{\Omega} f\left(d \alpha_{s}\right) \geq \int_{\Omega} f(d \alpha)
$$

Step 2. Let $\omega_{s}$ be a minimizing sequence of $\left(\mathcal{P}_{0}\right)$, i.e.

$$
\int_{\Omega} f\left(d \omega_{s}\right) \rightarrow m
$$

In view of the coercivity condition, we find that there exists a constant $c_{3}>0$ such that

$$
\left\|d \omega_{s}\right\|_{L^{p}} \leq c_{3}
$$

(i) According to Theorem 7.2 in [5] (when $p \geq 2$ ) and [17] (when $p>1$ ), we can find $\alpha_{s} \in \omega_{0}+W_{\delta, T}^{1, p}\left(\Omega ; \Lambda^{k-1}\right)$ such that

$$
\left\{\begin{array}{cl}
d \alpha_{s}=d \omega_{s} & \text { in } \Omega \\
\delta \alpha_{s}=0 & \text { in } \Omega \\
\nu \wedge \alpha_{s}=\nu \wedge \omega_{s}=\nu \wedge \omega_{0} & \text { on } \partial \Omega
\end{array}\right.
$$


and there exist constants $c_{4}, c_{5}>0$ such that

$$
\left\|\alpha_{s}\right\|_{W^{1, p}} \leq c_{4}\left[\left\|d \omega_{s}\right\|_{L^{p}}+\left\|\omega_{0}\right\|_{W^{1, p}}\right] \leq c_{5} .
$$

(ii) Therefore, up to the extraction of a subsequence that we do not relabel, there exists $\alpha \in \omega_{0}+W_{\delta, T}^{1, p}\left(\Omega ; \Lambda^{k-1}\right)$ such that

$$
\alpha_{s} \rightarrow \alpha, \quad \text { in } W^{1, p}\left(\Omega ; \Lambda^{k-1}\right) .
$$

(iii) We then use Theorem 8.16 in [5] (when $p \geq 2$ ) and [17] (when $p>1$ ), to find $\omega \in \omega_{0}+W_{0}^{1, p}\left(\Omega ; \Lambda^{k-1}\right)$ such that

$$
\left\{\begin{array}{cl}
d \omega=d \alpha & \text { in } \Omega \\
\omega=\omega_{0} & \text { on } \partial \Omega .
\end{array}\right.
$$

Step 3. We combine the two steps to get

$$
m=\liminf _{s \rightarrow \infty} \int_{\Omega} f\left(d \omega_{s}\right)=\liminf _{s \rightarrow \infty} \int_{\Omega} f\left(d \alpha_{s}\right) \geq \int_{\Omega} f(d \alpha)=\int_{\Omega} f(d \omega) \geq m .
$$

This concludes the proof of the theorem.

\section{Notations}

We gather here the notations which we will use throughout this article. For more details on exterior algebra and differential forms, see [5] and for the notions of convexity used in the calculus of variations, see [8].

1. Let $k, n$ be two integers.

- We write $\Lambda^{k}\left(\mathbb{R}^{n}\right)$ (or simply $\Lambda^{k}$ ) to denote the vector space of all alternating $k$-linear maps $f: \underbrace{\mathbb{R}^{n} \times \cdots \times \mathbb{R}^{n}}_{k \text {-times }} \rightarrow \mathbb{R}$. For $k=0$, we set $\Lambda^{0}\left(\mathbb{R}^{n}\right)=\mathbb{R}$. Note that $\Lambda^{k}\left(\mathbb{R}^{n}\right)=\{0\}$ for $k>n$ and, for $k \leq n, \operatorname{dim}\left(\Lambda^{k}\left(\mathbb{R}^{n}\right)\right)=\left(\begin{array}{l}n \\ k\end{array}\right)$.

- $\wedge,\lrcorner,\langle;\rangle$ and $*$ denote the exterior product, the interior product, the scalar product and the Hodge star operator respectively.

- If $\left\{e^{1}, \cdots, e^{n}\right\}$ is a basis of $\mathbb{R}^{n}$, then, identifying $\Lambda^{1}$ with $\mathbb{R}^{n}$,

$$
\left\{e^{i_{1}} \wedge \cdots \wedge e^{i_{k}}: 1 \leq i_{1}<\cdots<i_{k} \leq n\right\}
$$

is a basis of $\Lambda^{k}$. An element $\xi \in \Lambda^{k}\left(\mathbb{R}^{n}\right)$ will therefore be written as

$$
\xi=\sum_{1 \leq i_{1}<\cdots<i_{k} \leq n} \xi_{i_{1} i_{2} \cdots i_{k}} e^{i_{1}} \wedge \cdots \wedge e^{i_{k}}=\sum_{I \in \mathcal{T}_{k}^{n}} \xi_{I} e^{I},
$$

where

$$
\mathcal{T}_{k}^{n}=\left\{I=\left(i_{1}, \cdots, i_{k}\right) \in \mathbb{N}^{k}: 1 \leq i_{1}<\cdots<i_{k} \leq n\right\} .
$$


- We write

$$
e^{i_{1}} \wedge \cdots \wedge \widehat{e^{i_{s}}} \wedge \cdots \wedge e^{i_{k}}=e^{i_{1}} \wedge \cdots \wedge e^{i_{s-1}} \wedge e^{i_{s+1}} \wedge \cdots \wedge e^{i_{k}} .
$$

2. Let $\Omega \subset \mathbb{R}^{n}$ be a bounded open set.

- The spaces $C^{1}\left(\Omega ; \Lambda^{k}\right), W^{1, p}\left(\Omega ; \Lambda^{k}\right)$ and $W_{0}^{1, p}\left(\Omega ; \Lambda^{k}\right), 1 \leq p \leq \infty$ are defined in the usual way.

- For $\omega \in W^{1, p}\left(\Omega ; \Lambda^{k}\right)$, the exterior derivative $d \omega$ belongs to $L^{p}\left(\Omega ; \Lambda^{k+1}\right)$ and is defined by

$$
(d \omega)_{i_{1} \cdots i_{k+1}}=\sum_{j=1}^{k+1}(-1)^{j+1} \frac{\partial \omega_{i_{1} \cdots i_{j-1} i_{j+1} \cdots i_{k+1}}}{\partial x_{i_{j}}}
$$

for $1 \leq i_{1}<\cdots<i_{k+1} \leq n$. If $k=0$, then $d \omega \simeq \operatorname{grad} \omega$. If $k=1$, for $1 \leq i<j \leq n$

$$
(d \omega)_{i j}=\frac{\partial \omega_{j}}{\partial x_{i}}-\frac{\partial \omega_{i}}{\partial x_{j}}
$$

i.e. $d \omega \simeq \operatorname{curl} \omega$.

- The interior derivative (or codifferential) of $\omega \in W^{1, p}\left(\Omega ; \Lambda^{k}\right)$, denoted $\delta \omega$, belongs to $L^{p}\left(\Omega ; \Lambda^{k-1}\right)$ and is defined as

$$
\delta \omega=(-1)^{n(k-1)} *(d(* \omega)) .
$$

Acknowledgments. Part of this work was completed during visits of S. Bandyopadhyay to EPFL, whose hospitality and support is gratefully acknowledged. The research of S. Bandyopadhyay was partially supported by a SERB research project titled "Pullback Equation for Differential Forms".

\section{References}

[1] Bandyopadhyay S., Barroso A.C., Dacorogna B. and Matias J., Differential inclusions for differential forms, Calc. Var. Partial Differential Equations, 28 (2007), 449-469.

[2] Bandyopadhyay S., Dacorogna B. and Kneuss O., Some new results on differential inclusions for differential forms, to appear in Trans. Amer. Math. Soc. (2013).

[3] Bandyopadhyay S. and Sil S., Exterior convexity and classical calculus of variations, to appear in ESAIM Control Optim. Calc. Var. (2015).

[4] Bandyopadhyay S. and Sil S., In preparation. 
[5] Csató G., Dacorogna B. and Kneuss O., The pullback equation for differential forms, Birkhäuser/Springer, New York, 2012.

[6] Dacorogna B., Quasi-convexité et semi-continuité inférieure faible des fonctionnelles non linéaires, Ann. Scuola Norm. Sup. Pisa Cl. Sci. 9 (1982), 627-644.

[7] Dacorogna B., Weak continuity and weak lower semicontinuity of nonlinear functionals, Lecture Notes in Mathematics, 922. Springer-Verlag, Berlin-New York, 1982.

[8] Dacorogna B., Direct methods in the calculus of variations, second edition, Springer-Verlag, Berlin, 2007.

[9] Dacorogna B. and Fonseca I., A-B quasiconvexity and implicit partial differential equations, Calc. Var. Partial Differential Equations 14 (2002), 115-149.

[10] Fonseca I. and Müller S., A -quasiconvexity, lower semicontinuity, and Young measures, SIAM J. Math. Anal. 30 (1999), 1355-1390.

[11] Giannetti F. and Verde A., Variational integrals for elliptic complexes, Studia Math., vol. 140, (2000), 79-98.

[12] Marcellini P., Quasiconvex quadratic forms in two dimensions, Appl. Math. Optim. 11 (1984), 183-189.

[13] Morrey C.B., Multiple integrals in the calculus of variations, Springer-Verlag, Berlin, 1966.

[14] Murat F., Compacité par compensation, Ann. Scuola Norm. Sup. Pisa Cl. Sci. 5 (1978), 489-507.

[15] Robbin J.W., Rogers R.C. and Temple B., On weak continuity and the Hodge decomposition, Trans. Amer. Math. Soc. 303 (1987), 609-618.

[16] Serre D., Formes quadratiques et calcul des variations, J. Math. Pures Appl. 62 (1983), 117-196.

[17] Sil S., Ph.D. thesis.

[18] Stroffolini B. and Verde A., $X$-quasiconvexity in Carnot groups and lower semicontinuity results, Houston J. Math., vol. 35, (2009), 975-990.

[19] Sverak V., Quasiconvex functions with subquadratic growth, Proc. Roy. Soc. London Ser. A 433 (1991), 723-725. 
[20] Sverak V., Rank one convexity does not imply quasiconvexity, Proc. Roy. Soc. Edinburgh Sect. A 120 (1992), 185-189.

[21] Tartar L., Compensated compactness and applications to partial differential equations, in: Nonlinear analysis and mechanics, Proceedings, edited by Knops R.J., Res. Notes, vol. 39. Pitman, London, 1978, 136-212.

[22] Terpstra F.J., Die Darstellung biquadratischer Formen als Summen von Quadraten mit Anwendung auf die Variationsrechnung, Math. Ann. 116 (1939), 166-180. 